\title{
Deep Factorisation of the Stable Process III: the View from Radial Excursion Theory and the Point of Closest Reach
}

\author{
Andreas E. Kyprianou ${ }^{1}$ (D) . Victor Rivero ${ }^{2}$. Weerapat Satitkanitkul ${ }^{1,3}$
}

Received: 7 May 2018 / Accepted: 18 October 2019 / Published online: 16 November 2019

(C) The Author(s) 2019

\begin{abstract}
We compute explicitly the distribution of the point of closest reach to the origin in the path of any $d$-dimensional isotropic stable process, with $d \geq 2$. Moreover, we develop a new radial excursion theory, from which we push the classical Blumenthal-Getoor-Ray identities for first entry/exit into a ball (cf. Blumenthal et al. Trans. Amer. Math. Soc., 99, 540-554 1961) into the more complex setting of $n$-tuple laws for overshoots and undershoots. We identify explicitly the stationary distribution of any $d$-dimensional isotropic stable process when reflected in its running radial supremum. Finally, for such processes, and as consequence of some of the analysis of the aforesaid, we provide a representation of the Wiener-Hopf factorisation of the MAP that underlies the stable process through the Lamperti-Kiu transform. Our analysis continues in the spirit of Kyprianou (Ann. Appl. Probab., 20(2), 522-564 2010) and Kyprianou et al. (2015) in that our methodology is largely based around treating stable processes as self-similar Markov processes and, accordingly, taking advantage of their Lamperti-Kiu decomposition.
\end{abstract}

Keywords Stable processes $\cdot$ Lévy processes $\cdot$ Excursion theory $\cdot$ Riesz-Bogdan-Żak transform $\cdot$ Lamperti-Kiu transform

Mathematics Subject Classification (2010) Primary: 60G18 - 60G52 ; Secondary: 60G51

Victor Rivero was supported by EPSRC grant EP/M001784/1.

Andreas E. Kyprianou was supported by EPSRC grant EP/L002442/1 and EP/M001784/1

Andreas E. Kyprianou

a.kyprianou@bath.ac.uk

Victor Rivero

rivero@cimat.mx

Weerapat Satitkanitkul

weerapat.satit@gmail.com

1 Department of Mathematical Sciences, University of Bath, Claverton Down, Bath, BA2 7AY, UK

2 CIMAT A. C., Calle Jalisco s/n, Col. Valenciana, A. P. 402, C.P. 36000, Guanajuato, Gto., Mexico

3 Faculté des Sciences Bâtiment I, Département de mathématiques, Université d'Angers, 2

Boulevard Lavoisier, 49045 Angers cedex 01, France 


\section{Introduction and Main Results}

For $d \geq 1$, let $X:=\left(X_{t}: t \geq 0\right)$, with probabilities $\mathbb{P}_{x}, x \in \mathbb{R}^{d}$, be a $d$-dimensional isotropic stable process of index $\alpha \in(0,2)$. That is to say that $X$ is a $\mathbb{R}^{d}$-valued Lévy process having characteristic triplet $(0,0, \Pi)$, where

$$
\Pi(B)=\frac{2^{\alpha} \Gamma((d+\alpha) / 2)}{\pi^{d / 2}|\Gamma(-\alpha / 2)|} \int_{B} \frac{1}{|y|^{\alpha+d}} \mathrm{~d} y, \quad B \in \mathcal{B}(\mathbb{R}) .
$$

Equivalently, this means $X$ is a $d$-dimensional Lévy process with characteristic exponent $\Psi(\theta)=-\log \mathbb{E}_{0}\left(\mathrm{e}^{\mathrm{i} \theta X_{1}}\right)$ which satisfies

$$
\Psi(\theta)=|\theta|^{\alpha}, \quad \theta \in \mathbb{R} .
$$

Stable processes are also self-similar in the sense that they satisfy a scaling property. More precisely, for $c>0$ and $x \in \mathbb{R}^{d} \backslash\{0\}$,

$$
\text { under } \mathbb{P}_{x} \text {, the law of }\left(c X_{c^{-\alpha} t}, t \geq 0\right) \text { is equal to } \mathbb{P}_{c x} \text {. }
$$

As such, stable processes are useful for the study of the class of general Lévy processes and, more recently, for the study of the class of self-similar Markov processes. The latter are Feller processses which respect the scaling relation (1.2). Accordingly it is said that they are self-similar with index $1 / \alpha$.

In the last few years, the fluctuation theory of one-dimensional stable processes has benefitted from the interplay between the general theory of Lévy processes and the general theory of self-similar Markov processes. Examples of recent results include more general distributional identities of the first passage problem for the half-line in one dimension, cf. [12], the distribution of the first point of entry into a strip, [13], and the stationary distribution of the process reflected in its radial maximum, [14].

In this spirit, we aim to add to this list of new fluctuation identities but now the setting of isotropic stable processes in dimension $d \geq 2$ (henceforth assumed). Such processes are transient in the sense that

$$
\lim _{t \rightarrow \infty}\left|X_{t}\right|=\infty
$$

and do not hit points almost surely. Accordingly, when issued from a point $x \neq 0$, it makes sense to define the point of closest reach to the origin; that is, the coordinates of the point in the closure of the range of $X$ with minimal radial distance from the origin. Our main results offer the exact distribution for the point of closest reach as well as a number of completely new fluctuation identities that fall out of its proof and the use of radial excursion theory.

Before describing them in more detail, let us define point of closest reach with a little more precision. We need to note a number of facts. First, isotropy and transience ensures that $|X|$ is a conservative positive self-similar Markov process with index of self-similarity $1 / \alpha$. Accordingly it can be represented via the classical Lamperti transformation

$$
\left|X_{t}\right|=\mathrm{e}^{\xi_{\varphi(t)}}, \quad t \geq 0,
$$

where

$$
\varphi(t)=\inf \left\{s>0: \int_{0}^{s} \mathrm{e}^{\alpha \xi_{u}} \mathrm{~d} u>t\right\}
$$

and $\xi=\left(\xi_{s}: s \geq 0\right)$, with probabilities $\mathbf{P}_{x}, x \in \mathbb{R}$, is a one-dimensional Lévy process. It was shown in [5] that the process $\xi$ belongs to the class of so-called hypergeometric Lévy processes. In particular, its Wiener-Hopf factorisation is explicit. Indeed, suppose we write 
its characteristic exponent $\Psi_{\xi}(\theta)=-\log \mathbf{E}_{0}\left[\exp \left\{\mathrm{i} \theta \xi_{1}\right\}\right], \theta \in \mathbb{R}$, then up to a multiplicative constant,

$$
\Psi_{\xi}(\theta)=\frac{\Gamma\left(\frac{1}{2}(-\mathrm{i} \theta+\alpha)\right)}{\Gamma\left(-\frac{1}{2} \mathrm{i} \theta\right)} \times \frac{\Gamma\left(\frac{1}{2}(\mathrm{i} \theta+d)\right)}{\Gamma\left(\frac{1}{2}(\mathrm{i} \theta+d-\alpha)\right)}, \quad \theta \in \mathbb{R},
$$

where the two terms either side of the multiplication sign constitute the two Wiener-Hopf factors. See e.g. Chapter VI in [2] for background. Recall that if $\Psi$ is the characteristic exponent of any Lévy process, say $Z$, then there exist two Bernstein functions $\kappa$ and $\hat{\kappa}$ (see [19] for a definition) such that, up to a multiplicative constant,

$$
\Psi(\theta)=\kappa(-\mathrm{i} \theta) \hat{\kappa}(\mathrm{i} \theta), \quad \theta \in \mathbb{R} .
$$

Identity (1.7) is what we refer to as the Wiener-Hopf factorisation. The left-hand factor characterises the distribution of the range of the running maximum and the right-hand factor, the range of the running infimum of $Z$. It can be checked that both belong to the class of socalled beta subordinators (see [8], as well as some of the discussion later in this paper) and, in particular, have infinite activity. The later implies that $\xi$ is regular for both the upper and lower half-lines (meaning that, when issued at the origin, the process $\xi$ will instantaneously visit both the upper and lower half-line with probability 1; see e.g. Definition 6.4 of [10]), which in turn, thanks to Eq. 1.4, noting the continuity and strictly increasing nature of Eq. 1.5, means that any sphere of radius $r>0$ is regular for both its interior and exterior for $X$ (meaning that, when issued anywhere on the sphere or radius $r$, the process $X$ will visit both the interior and exterior of the sphere instantaneously with probability 1 ). This and the fact that $X$ has càdlàg paths ensures that, denoting

$$
\mathrm{G}(t):=\sup \left\{s \leq t:\left|X_{s}\right|=\inf _{u \leq t}\left|X_{u}\right|\right\}=\sup \left\{s \leq t:\left|X_{s}\right|=\inf _{u \leq s}\left|X_{u}\right|\right\}, \quad t \geq 0,
$$

the quantity $X_{\mathrm{G}(t)}$ is well defined as the point of closest reach to the origin up to time $t$ in the sense that $X_{\mathrm{G}(t)-}=X_{\mathrm{G}(t)}$ and

$$
\left|X_{\mathrm{G}(t)}\right|=\inf _{s \leq t}\left|X_{s}\right| .
$$

The process $(\mathrm{G}(t), t \geq 0)$ is monotone increasing and hence $\mathrm{G}(\infty)=\lim _{t \rightarrow \infty} \mathrm{G}(t)$ exists almost surely. Moreover, as $X$ is transient in the sense of Eq. 1.3, it is also clear that, almost surely, $\mathrm{G}(\infty)=\mathrm{G}(t)$ for all $t$ sufficiently large and that

$$
\left|X_{\mathrm{G}(\infty)}\right|=\inf _{s \geq 0}\left|X_{s}\right| .
$$

Our first main result provides explicitly the law of $X_{\mathrm{G}(\infty)}$.

Theorem 1.1 (Point of Closest Reach to the origin) The law of the point of closest reach to the origin is given by

$$
\mathbb{P}_{x}\left(X_{G(\infty)} \in \mathrm{d} y\right)=\pi^{-d / 2} \frac{\Gamma(d / 2)^{2}}{\Gamma((d-\alpha) / 2) \Gamma(\alpha / 2)} \frac{\left(|x|^{2}-|y|^{2}\right)^{\alpha / 2}}{|x-y|^{d}|y|^{\alpha}} \mathrm{d} y, \quad 0<|y|<|x| .
$$

Fundamentally, the proof of Theorem 1.1 will be derived from two main facts. The first is a suite of exit/entrance formulae from balls for stable processes which come from the classical work of Blumenthal-Getoor-Ray [3]. To state these results, let us write

$$
\tau_{r}^{\oplus}=\inf \left\{t>0:\left|X_{t}\right|<r\right\} \text { and } \tau_{r}^{\ominus}=\inf \left\{t>0:\left|X_{t}\right|>r\right\},
$$

for $r>0$. 
Theorem 1.2 (Blumenthal-Getoor-Ray [3]) For either $|x|<r<|y|$ when $\tau=\tau_{r}^{\ominus}$, or $|y|<r<|x|$ when $\tau=\tau_{r}^{\oplus}$,

$$
\mathbb{P}_{x}\left(X_{\tau} \in \mathrm{d} y\right)=C_{\alpha, d} \frac{\left.\left.\left|r^{2}-\right| x\right|^{2}\right|^{\alpha / 2}}{\left.\left.\left|r^{2}-\right| y\right|^{2}\right|^{\alpha / 2}}|x-y|^{-d} \mathrm{~d} y,
$$

where $C_{\alpha, d}=\pi^{-(d / 2+1)} \Gamma(d / 2) \sin (\pi \alpha / 2)$. Moreover, for $|x|>r$,

$$
\mathbb{P}_{x}\left(\tau_{r}^{\oplus}=\infty\right)=\frac{\Gamma(d / 2)}{\Gamma((d-\alpha) / 2) \Gamma(\alpha / 2)} \int_{0}^{\left(|x|^{2} / r^{2}\right)-1}(u+1)^{-d / 2} u^{\alpha / 2-1} \mathrm{~d} u
$$

and, for $|x|<r$ and bounded measurable $f$ on $\mathbb{R}^{d}$,

$$
\mathbb{E}_{x}\left[\int_{0}^{\tau_{r}^{\ominus}} f\left(X_{s}\right) \mathrm{d} s\right]=\int_{|y|>r} h_{r}^{\ominus}(x, y) f(y) \mathrm{d} y
$$

such that

$$
h_{r}^{\ominus}(x, y)=2^{-\alpha} \pi^{-d / 2} \frac{\Gamma(d / 2)}{\Gamma(\alpha / 2)^{2}}|x-y|^{\alpha-d} \int_{0}^{\zeta_{r}(x, y)}(u+1)^{-d / 2} u^{\alpha / 2-1} \mathrm{~d} u, \quad|y|<r,
$$

where $\zeta_{r}^{\ominus}(x, y)=\left(r^{2}-|x|^{2}\right)\left(r^{2}-|y|^{2}\right) / r^{2}|x-y|^{2}$.

Remark 1.1 It is worth remarking that Eq. 1.9 can be used to derive the density of $\left|X_{\mathrm{G}(\infty)}\right|$ quite easily. Indeed, thanks to the scaling property and rotational symmetry, it suffices in this respect to consider the law of $\left|X_{\mathrm{G}(\infty)}\right|$ under $\mathbb{P}_{1}$, where $1=(1,0, \cdots, 0)$ is the 'North Pole' on $\mathbb{S}^{d-1}$. In this respect, we note that $\mathbb{P}_{1}\left(\left|X_{\mathrm{G}(\infty)}\right| \leq r\right)=1-\mathbb{P}_{1}\left(\tau_{r}^{\oplus}=\infty\right)$, hence, for $\gamma>0$,

$$
\begin{aligned}
\mathbb{E}_{1}\left[\left|X_{\mathrm{G}(\infty)}\right|^{2 \gamma}\right] & =\int_{0}^{1} r^{2 \gamma} \mathrm{dP}_{1}\left(\left|X_{\mathrm{G}(\infty)}\right| \leq r\right) \\
& =\frac{2 \Gamma(d / 2)}{\Gamma((d-\alpha) / 2) \Gamma(\alpha / 2)} \int_{0}^{1} r^{2 \gamma+(d-\alpha)-1}\left(1-r^{2}\right)^{\frac{\alpha}{2}-1} \mathrm{~d} r \\
& =\frac{\Gamma(d / 2)}{\Gamma((d-\alpha) / 2) \Gamma(\alpha / 2)} \int_{0}^{1} u^{\gamma+\frac{(d-\alpha)}{2}-1}(1-u)^{\frac{\alpha}{2}-1} \mathrm{~d} u .
\end{aligned}
$$

From this it is straightforward to see that $\left|X_{\mathrm{G}(\infty)}\right|$ under $\mathbb{P}_{1}$ is equal in law to $\sqrt{\AA}$, where $\mathrm{A}$ is a $\operatorname{Beta}((d-\alpha) / 2, \alpha / 2)$ distribution.

The second main fact that drives the proof of Theorem 1.1 is the Lamperti-Kiu representation of self-similar Markov processes. To describe it, we need to introduce the notion of a Markov Additive Process, henceforth written MAP for short.

Let $\mathbb{S}^{d-1}=\left\{x \in \mathbb{R}^{d}:|x|=1\right\}$. With an abuse of previous notation, we say that $(\Xi, \Upsilon)=\left(\left(\Xi_{t}, \Upsilon_{t}\right), t \geq 0\right)$ is a MAP if it is Feller process on $\mathbb{R} \times \mathbb{S}^{d-1}$, with probabilities $\mathrm{P}_{x, \theta}, x \in \mathbb{R}^{d}, \theta \in \mathbb{S}^{\bar{d}-1}$, such that, for any $t \geq 0$, the conditional law of the process $\left(\left(\Xi_{s+t}-\Xi_{t}, \Upsilon_{s+t}\right): s \geq 0\right)$, given $\left(\left(\Xi_{u}, \Upsilon_{u}\right), u \leq t\right)$, is that of $(\Xi, \Upsilon)$ under $\mathrm{P}_{0, \theta}$, with $\theta=\Upsilon_{t}$. For a MAP pair $\left(\left(\Xi_{u}, \Upsilon_{u}\right), u \leq t\right)$, we call $\Xi$ the ordinate and $\Upsilon$ the modulator.

According to one of the main results in [1], there exists a MAP, which we will henceforth write as $(\xi, \Theta)$ with probabilities $\mathbf{P}_{x, \theta}, x \in \mathbb{R}^{d}, \theta \in \mathbb{S}^{d-1}$ such that the $d$-dimensional isotropic stable process can be written

$$
X_{t}=\exp \left\{\xi_{\varphi(t)}\right\} \Theta_{\varphi(t)} \quad t \geq 0,
$$


where $\varphi$ has the same definition as Eq. 1.5. Note that $\xi$ was previously used to denote the underlying Lévy process for the Lamperti transform of $\left|X_{t}\right|$ in Eq. 1.4. This is not an abuse of notation as Eq. 1.11 must be consistent with Eq. 1.4. In this sense, we can unify our notation further by agreeing that e.g. $\mathbf{P}_{x}\left(\xi_{t} \in A\right)=\int_{\mathbb{S} d-1} \mathbf{P}_{x, \theta}\left(\xi_{t} \in A, \Theta_{t} \in \mathrm{d} \theta\right)$, for $t \geq 0$ and $A \in \mathcal{B}(\mathbb{R})$. Whilst the processes $\Theta$ and $\xi$ are corollated, the aforesaid remarks indicate the execptional property (as a relative statement to the case of a general MAP) that $\xi$ alone is a Markov process (in fact a Lévy process) in addition both $\Theta$ alone and $(\xi, \Theta)$ being Markovian. Moreover, it is clearly the case that $\Theta$ is isotropic in the distributional sense, and hence an ergodic process on a compact domain with uniform stationary distribution.

Remark 1.2 Noting that $X_{\mathrm{G}(\infty)}=\left|X_{\mathrm{G}(\infty)}\right| \times \arg \left(X_{\mathrm{G}(\infty)}\right)$, it is tempting to believe that it is a simple step to use the distributional identity in Remark 1.1 to build the law of $X_{\mathrm{G}(\infty)}$. This seems tempting because of the similarity between Eq. 1.8 and the a posteriori conclusion in Theorem 1.1. Indeed one of our approaches was to try to derive the one from the other by a simple limiting procedure. Making this idea rigorous turns out to be much more difficult than imagined on account of the subtle dependence between radial and angular behaviour of the MAP that underlies the stable process.

Our proof of Theorem 1.1 will take us on a journey through an excursion theory of $X$ from its radial maximum. In dimension $d \geq 2$, this is the first time, to our knowledge, that such a radial excursion theory has been used. See however [6] in which the basis of the analysis there can be interpreted as a radial excursion theory, albeit in one dimension. What makes radial excursion difficult over and above standard excursion theory is that it deals with excursions of the process $X_{t} / M_{t}, t \geq 0$, away from the set $\mathbb{S}^{d-1}$, where $M_{t}:=\sup _{s \leq t}\left|X_{s}\right|, t \geq 0$. As such one needs to work with a family of excursion measures that appear in the associated exit system, which are indexed by $\mathbb{S}^{d-1}$ (more precise details will be provided in due course).

The use of radial excursion theory also allow us to prove the following $n$-tuple laws at first entry/exit of a ball (i.e. the joint laws of the relevant quantities at first passage, see below), which provide a non-trivial extension to the classical identities of Blumenthal, Getoor and Ray [3] given in Theorem 1.2.

Theorem 1.3 (Triple law at first entrance/exit of a ball) Fix $r>0$ and define, for $x, z, y, v \in \mathbb{R}^{d} \backslash\{0\}$,

$$
\chi_{x}(z, y, v):=\pi^{-3 d / 2} \frac{\Gamma((d+\alpha) / 2)}{|\Gamma(-\alpha / 2)|} \frac{\Gamma(d / 2)^{2}}{\Gamma(\alpha / 2)^{2}} \frac{\left.|| z\right|^{2}-\left.\left.|x|^{2}\right|^{\alpha / 2}|| y\right|^{2}-\left.|z|^{2}\right|^{\alpha / 2}}{|z-x|^{d}|z-y|^{d}|v-y|^{\alpha+d}} .
$$

(i) Write

$$
G\left(\tau_{r}^{\oplus}\right)=\sup \left\{s<\tau_{r}^{\oplus}:\left|X_{s}\right|=\inf _{u \leq s}\left|X_{u}\right|\right\}
$$

for the instant of closest reach of the origin before first entry into $\mathbb{B}_{r}^{d}=\left\{x \in \mathbb{R}^{d}\right.$ : $|x| \leq r\}$. For $|x|>|z|>r,|y|>|z|$ and $|v|<r$,

$$
\mathbb{P}_{x}\left(X_{G\left(\tau_{r}^{\oplus}\right)} \in \mathrm{d} z, X_{\tau_{r}^{\oplus}-} \in \mathrm{d} y, X_{\tau_{r}^{\oplus}} \in \mathrm{d} v ; \tau_{r}^{\oplus}<\infty\right)=\chi_{x}(z, y, v) \mathrm{d} z \mathrm{~d} y \mathrm{~d} v .
$$

(ii) Define $\mathcal{G}(t)=\sup \left\{s<t:\left|X_{s}\right|=\sup _{u \leq s}\left|X_{u}\right|\right\}, t \geq 0$, and write

$$
\mathcal{G}\left(\tau_{r}^{\ominus}\right)=\sup \left\{s<\tau_{r}^{\ominus}:\left|X_{s}\right|=\sup _{u \leq s}\left|X_{u}\right|\right\}
$$


for the instant of furthest reach from the origin immediately before first exit from $\mathbb{B}_{r}^{d}$. For $|x|<|z|<r,|y|<|z|$ and $|v|>r$,

$$
\mathbb{P}_{x}\left(X_{\mathcal{G}\left(\tau_{r}^{\ominus}\right)} \in \mathrm{d} z, X_{\tau_{r}^{\ominus}-} \in \mathrm{d} y, X_{\tau_{r}^{\ominus}} \in \mathrm{d} v\right)=\chi_{x}(z, y, v) \mathrm{d} z \mathrm{~d} y \mathrm{~d} v .
$$

Marginalising the first triple law in Theorem 1.1 to give the joint law of the pair $\left(X_{\mathrm{G}\left(\tau_{r}^{\oplus}\right)}, X_{\tau_{r}^{\oplus}}\right)$ or the pair $\left(X_{\tau_{r}^{\oplus}-}, X_{\tau_{r}^{\oplus}}\right)$ is not necessarily straightforward (although the reader familiar with the manipulation of Riesz potentials may feel more comfortable as such). Whist an analytical computation for the marginalisation is, in principle, be possible, if not tedious, we provide a proof which combines other fluctuation identities that we will uncover en route.

Corollary 1.3 (First entrance/exit and closest reach) Fix $r>0$ and define, for $x, z, v \in$ $\mathbb{R}^{d} \backslash\{0\}$,

$$
\chi_{x}(z, \bullet, v):=\frac{\Gamma(d / 2)^{2}}{\pi^{d}|\Gamma(-\alpha / 2)| \Gamma(\alpha / 2)} \frac{\left.|| z\right|^{2}-\left.|x|^{2}\right|^{\alpha / 2}}{\left.|| z\right|^{2}-\left.|v|^{2}\right|^{\alpha / 2}|z-v|^{d}|z-x|^{d}} .
$$

(i) For $|x|>|z|>r,|v|<r$,

$$
\mathbb{P}_{x}\left(X_{G\left(\tau_{r}^{\oplus}\right)} \in \mathrm{d} z, X_{\tau_{r}^{\oplus}} \in \mathrm{d} v ; \tau_{r}^{\oplus}<\infty\right)=\chi_{x}(z, \bullet, v) \mathrm{d} z \mathrm{~d} v .
$$

(ii) For $|x|<|z|<r$ and $|v|>r$,

$$
\mathbb{P}_{x}\left(X_{\mathcal{G}\left(\tau_{r}^{\ominus}\right)} \in \mathrm{d} z, X_{\tau_{r}^{\ominus}} \in \mathrm{d} v\right)=\chi_{x}(z, \bullet, v) \mathrm{d} z \mathrm{~d} v .
$$

Corollary 1.4 (First entrance/exit and preceding position) Fix $r>0$ and define, for $x, z, y, v \in \mathbb{R}^{d} \backslash\{0\}$,

$\chi_{x}(\bullet, y, v):=\frac{\Gamma((d+\alpha) / 2) \Gamma(d / 2)}{\pi^{d}|\Gamma(-\alpha / 2)| \Gamma(\alpha / 2)^{2}}\left(\int_{0}^{\zeta_{r}^{\oplus}(x, y)}(u+1)^{-d / 2} u^{\alpha / 2-1} \mathrm{~d} u\right) \frac{|x-y|^{\alpha-d}}{|v-y|^{\alpha+d}} \mathrm{~d} v \mathrm{~d} y$,

where

$$
\zeta_{r}^{\oplus}(x, y):=\left(|x|^{2}-r^{2}\right)\left(|y|^{2}-r^{2}\right) / r^{2}|x-y|^{2} .
$$

(i) For $|x|,|y|>r,|v|<r$,

$$
\mathbb{P}_{x}\left(X_{\tau_{r}^{\oplus}-} \in \mathrm{d} y, X_{\tau_{r}^{\oplus}} \in \mathrm{d} v ; \tau_{r}^{\oplus}<\infty\right)=\chi_{x}(\bullet, y, v) \mathrm{d} y \mathrm{~d} v .
$$

(ii) For $|x|,|y|<r$ and $|v|>r$,

$$
\mathbb{P}_{x}\left(X_{\tau_{r}^{\ominus}-} \in \mathrm{d} y, X_{\tau_{r}^{\ominus}} \in \mathrm{d} v\right)=\chi_{x}(\bullet, y, v) \mathrm{d} y \mathrm{~d} v .
$$

In $[11,14]$, one-dimensional stable processes were considered (up to first hitting of the origin in the case that $\alpha \in(1,2))$, for which the process $\Theta$ in the underlying MAP is nothing more than a two-state Markov chain on $\{1,-1\}$. Such MAPs are known to have a Wiener-Hopf-type decomposition.

To be more precise, one may describe the semigroup of $(\xi, \Theta)$ via a matrix Laplace exponent which plays a similar role to the characteristic exponent of $\xi$. When it exists, the matrix $\Psi$, mapping $\mathbb{C}$ to the space of $2 \times 2$ complex valued matrices, ${ }^{1}$ satisfies,

$$
\left(e^{-\boldsymbol{\Psi}(z) t}\right)_{i, j}=\mathbf{E}_{0, i}\left[e^{-z \xi_{t}} ; \Theta_{t}=j\right], \quad i, j= \pm 1, t \geq 0 .
$$

${ }^{1}$ Here the matrix entries are arranged by

$$
A=\left(\begin{array}{cc}
A_{1,1} & A_{1,-1} \\
A_{-1,1} & A_{-1,-1}
\end{array}\right)
$$


In fact, it is known to take the form

$$
\boldsymbol{\Psi}(z)=\left(\begin{array}{cc}
\frac{\Gamma(\alpha+z) \Gamma(1-z)}{\Gamma(\alpha \hat{\rho}+z) \Gamma(1-\alpha \hat{\rho}-z)} & -\frac{\Gamma(\alpha+z) \Gamma(1-z)}{\Gamma(\alpha \hat{\rho}) \Gamma(1-\alpha \hat{\rho})} \\
-\frac{\Gamma(\alpha+z) \Gamma(1-z)}{\Gamma(\alpha \rho) \Gamma(1-\alpha \rho)} & \frac{\Gamma(\alpha+z) \Gamma(1-z)}{\Gamma(\alpha \rho+z) \Gamma(1-\alpha \rho-z)}
\end{array}\right),
$$

for $\operatorname{Re}(z) \in(-1, \alpha)$; see [7] and [9]. Similar to the case of Lévy processes, we can define $\boldsymbol{\kappa}$ and $\hat{\boldsymbol{\kappa}}$ as the matrix Laplace exponents of two MAPs, each with non-decreasing ordinate, whose ordinate ranges and accompanying modulation coincide in distribution with the the range of the running maximum of $\xi$ and that of the dual process $\hat{\xi}$, with accompanying modulation. The analogue of the Wiener-Hopf factorisation for MAPs states that, up to premultiplying $\boldsymbol{\kappa}$ or $\hat{\kappa}$ (and hence equivalently up to pre-multiplying $\Psi$ ) by a strictly positive diagonal matrix, we have that

$$
\boldsymbol{\Psi}(-\mathrm{i} \lambda)=\boldsymbol{\Delta}_{\pi}^{-1} \hat{\boldsymbol{\kappa}}(\mathrm{i} \lambda)^{T} \boldsymbol{\Delta}_{\pi} \boldsymbol{\kappa}(-\mathrm{i} \lambda),
$$

for $\lambda \in \mathbb{R}$, where

$$
\boldsymbol{\Delta}_{\pi}:=\left(\begin{array}{cc}
\sin (\pi \alpha \rho), & 0 \\
0 & \sin (\pi \alpha \hat{\rho})
\end{array}\right) .
$$

In the setting of the MAP which underlies the stable process, the so-called deep WienerHopf factorisation was computed in [11], thereby providing the first explicit example of the Wiener-Hopf factorisation for a MAP. When $X$ is a symmetric one-dimensional stable process, then, without loss of generality, we may take $\boldsymbol{\Delta}_{\pi}$ as the identity matrix, the underlying MAP becomes symmetric, in which case $\hat{\kappa}^{T}=\hat{\kappa}$ and, moreover, $\hat{\kappa}(\lambda)=\kappa(\lambda+1-\alpha)$, $\lambda \geq 0$. In that case, the factorisation simplifies to

$$
\boldsymbol{\Psi}(-\mathrm{i} \lambda)=\kappa(\mathrm{i} \lambda+1-\alpha) \kappa(-\mathrm{i} \lambda), \quad \lambda \in \mathbb{R},
$$

up to multiplication by a strictly positive diagonal matrix.

For dimension $d \geq 2$, by adopting the right mathematical language, we are also able to provide the deep factorisation of the $d$-dimensional isotropic stable process, which also generalises the situation in one dimension. To this end, let us introduce the notion of the descending ladder MAP process for $(\xi, \Theta)$.

It is not difficult to show that the pair $\left(\left(\bar{\xi}_{t}-\xi_{t}, \Theta_{t}\right), t \geq 0\right)$, forms a strong Markov process, where $\bar{\xi}_{t}:=\sup _{s \leq t} \xi_{s}, t \geq 0$ is the running maximum of $\xi$. Naturally, on account of the fact that $\xi$, as a lone process, is a Lévy process, $\left(\bar{\xi}_{t}-\xi_{t}, t \geq 0\right)$, is also a strong Markov process, but we are more interested here on its dependency on $\Theta$. Suppose we denote by $L$ the local time at zero of $\bar{\xi}-\xi$, then we can introduce the following processes

$$
H_{t}^{+}=\xi_{L_{t}^{-1}} \text { and } \Theta_{t}^{+}=\Theta_{L_{t}^{-1}}, \quad t \geq 0 .
$$

The strong Markov property tells us that $\left(L_{t}^{-1}, H_{t}^{+}, \Theta_{t}^{+}\right), t \geq 0$, defines a Markov additive process, whose first two elements are ordinates that are non-decreasing. In this sense, $L$ also serves as a local time on the set $\{0\} \times \mathbb{S}^{d-1}$ of the Markov process $(\bar{\xi}-\xi, \Theta)$. Because $\xi$, alone, is also a Lévy process then the pair $\left(L^{-1}, H^{+}\right)$, without reference to the associated modulation $\Theta^{+}$, are Markovian and play the role of the ascending ladder time and height subordinators of $\xi$.

If we are to state a factorisation analogous to Eq. 1.14, we must understand how to define the quantities that are analogous to $\boldsymbol{\Psi}$ and $\boldsymbol{\kappa}$. Inspiration to this end comes from [14], where it was shown that it is more convenient to understand the relationship (1.13) in its inverse 
form. This is equivalent to showing how the resolvent of the underlying MAP relates to the potential measures associated to $\boldsymbol{\kappa}$ and $\hat{\boldsymbol{\kappa}}$.

Therefore, in the current setting of $d$-dimensional isotropic stable processes, we define the operators

$$
\mathbf{R}_{z}[f](\theta)=\mathbf{E}_{0, \theta}\left[\int_{0}^{\infty} \mathrm{e}^{-z \xi t} f\left(\Theta_{t}\right) \mathrm{d} t\right], \quad \theta \in \mathbb{S}^{d-1}, z \in \mathbb{C}, \operatorname{Re}(z) \geq 0 .
$$

and

$$
\boldsymbol{\rho}_{z}[f](\theta)=\mathbf{E}_{0, \theta}\left[\int_{0}^{\infty} \mathrm{e}^{-z H_{t}^{+}} f\left(\Theta_{t}^{+}\right) \mathrm{d} t\right], \quad \theta \in \mathbb{S}^{d-1}, z \in \mathbb{C}, \operatorname{Re}(z) \geq 0 .
$$

for bounded measurable $f: \mathbb{S}^{d-1} \mapsto[0, \infty)$. Note, by Eq. 1.6, both $\xi$ and $H^{+}$both have finite mean and hence grow at linearly by the strong law of large numbers. Accordingly the integrals (1.15) and (1.16) are bounded for the aforesaid family of functions.

Theorem 1.4 (Deep factorisation of the $d$-dimensional isotropic stable process) Suppose that $f: \mathbb{S}^{d-1} \mapsto[0, \infty)$ is bounded and measurable. Then

$$
\boldsymbol{R}_{c-\mathrm{i} \lambda}[f](\theta)=C_{\alpha, d} \boldsymbol{\rho}_{\mathrm{i} \lambda+d-\alpha-c}\left[\boldsymbol{\rho}_{c-\mathrm{i} \lambda}[f]\right](\theta), \quad \theta \in \mathbb{S}^{d-1}, \lambda \in \mathbb{R}, c \in(0, d-\alpha),
$$

where $C_{\alpha, d}=2^{-\alpha} \Gamma((d-\alpha) / 2)^{2} / \Gamma(d / 2)^{2}$. Moreover,

$$
\rho_{z}[f](\theta)=\frac{\pi^{-d / 2} \Gamma(d / 2)^{2}}{\Gamma((d-\alpha) / 2) \Gamma(\alpha / 2)} \int_{|y|>1} f(\arg (y)) \frac{\left.|| y\right|^{2}-\left.|\theta|^{2}\right|^{\alpha / 2}}{|y|^{\alpha+z}|\theta-y|^{d}} \mathrm{~d} y, \quad \operatorname{Re}(z)>0
$$

and

$$
\boldsymbol{R}_{z}[f](\theta)=\frac{\Gamma((d-\alpha) / 2)}{2^{\alpha} \pi^{d / 2} \Gamma(\alpha / 2)} \int_{\mathbb{R}^{d}} f(\arg (y))|y|^{-z-\alpha}|y-\theta|^{\alpha-d} \mathrm{~d} y, \quad \operatorname{Re}(z) \in(0, d-\alpha) .
$$

This, our third main result, is the first example we know of in the literature which provides in explicit detail the Wiener-Hopf factorisation of a MAP for which the modulator has an uncountable state space.

Remark 1.5 The reader is reminded that Theorem 1.4 is stated for dimension $d \geq 2$ and for isotropic processes. However, if we are careful about assumptions, it is easily verified that the theorem also holds in dimension $d=1$. Recall that that in dimension $d \geq 2$ we have $\lim _{t \rightarrow \infty}\left|X_{t}\right|=\infty$ almost surely. This is a crucial part of the proof of Theorem 1.4. Accordingly when checking the proof in dimension $d=1$, we should confine ourselves to the additional assumptions that the process is symmetric and $\alpha \in(0,1)$.

The aforementioned case was discussed in [14]. In that case, $\Theta$ and $\Theta^{+}$live on the states $\{-1,1\}$ and hence, in the definitions (1.15) and (1.16), we have $f:\{1,-1\} \mapsto[0, \infty)$. In effect we can thus reduce the resolvents (1.15) and (1.16) to matrices. Accordingly we prefer to write (for all appropriate $z \in \mathbb{C}$ ), $\mathbf{R}_{z}(j, k)=\mathbf{R}_{z}\left[\mathbf{1}_{k}\right](j)$ and $\boldsymbol{\rho}_{z}\left[\mathbf{1}_{k}\right](j)=\boldsymbol{\rho}_{z}(j, k)$, 
for $j, k \in\{1,-1\}$, where $\mathbf{1}_{k}=\mathbf{1}_{k}(\cdot)$ is the indicator function of the state $k$. The factorisation (1.17) now reads (up to an unimportant multiplicative constant)

$$
\begin{aligned}
\mathbf{R}_{c-\mathrm{i} \lambda}(j, k) & =\boldsymbol{\rho}_{\mathrm{i} \lambda+d-\alpha-c}\left[\boldsymbol{\rho}_{c-\mathrm{i} \lambda}\left[\mathbf{1}_{k}\right]\right](j) \\
& =\sum_{\ell=1,-1} \mathbf{E}_{0, j}\left[\int_{0}^{\infty} \mathrm{e}^{-(\mathrm{i} \lambda+d-\alpha-c) H_{t}^{+}} \mathbf{1}_{\ell}\left(\Theta_{t}^{+}\right) \mathrm{d} t\right] \boldsymbol{\rho}_{c-\mathrm{i} \lambda}\left[\mathbf{1}_{k}\right](\ell) \\
& =\sum_{\ell=1,-1} \boldsymbol{\rho}_{\mathrm{i} \lambda+d-\alpha-c}(j, \ell) \boldsymbol{\rho}_{c-\mathrm{i} \lambda}(\ell, k), \quad k, \ell \in\{1,-1\} .
\end{aligned}
$$

In the same notation introduced on p349 of [15], we have e.g. that

$\boldsymbol{\rho}_{c-\mathrm{i} \lambda}(j, k)=\mathbf{E}_{0, j}\left[\int_{0}^{\infty} \mathrm{e}^{-(c-\mathrm{i} \lambda) H_{t}^{+}} \mathbf{1}_{k}\left(\Theta_{t}^{+}\right) \mathrm{d} t\right]=\int_{0}^{\infty} \mathrm{e}^{-(c-\mathrm{i} \lambda) y} \boldsymbol{u}_{j, k}(y) \mathrm{d} y:=\boldsymbol{\kappa}_{i, j}^{-1}(c-\mathrm{i} \lambda)$,

where $\left(\boldsymbol{u}_{j, k}, j, k \in\{1,-1\}\right)$ is the potential density of $\left(H^{+}, \Theta^{+}\right)$. We can also easily check that $\operatorname{det}(\Psi(c-\mathrm{i} \lambda)) \neq 0$ and hence $\Psi(c-\mathrm{i} \lambda)$ is invertible. It follows by consolidating (1.20) with the matrix Wiener-Hopf factorisation (1.14), by exchanging the sign of $\lambda$ and performing an analytical extension of the identity, that $\mathbf{R}_{c-\mathrm{i} \lambda}(j, k)=\left[\boldsymbol{\Psi}^{-1}(1-\alpha-c+\right.$ i入) $]_{j, k}$.

Our final main result concerns the stationary distribution of the stable process reflected in its radial supremum. Define $M_{t}=\sup _{s \leq t}\left|X_{s}\right|, t \geq 0$. It is a straightforward computation to show that $\left(X_{t} / M_{t}, M_{t}\right), t \geq 0$ is a Markov process which lives on $\mathbb{B}_{d} \times(0, \infty)$, where $\mathbb{B}_{d}=\left\{x \in \mathbb{R}^{d}:|x| \leq 1\right\}$. Thanks to the transience of $X$, it is clear that $\lim _{t \rightarrow \infty} M_{t}=\infty$, however, thanks to repeated normalisation of $X$ by its radial maximum, we can expect that the $\lim _{t \rightarrow \infty} X_{t} / M_{t}$ exists in distribution. Indeed, in the one-dimensional setting this has already been proved to be the case in [14].

Theorem 1.5 For all bounded measurable $f: \mathbb{B}_{d} \mapsto \mathbb{R}$ and $x \in \mathbb{R} \backslash\{0\}$

$$
\lim _{t \rightarrow \infty} \mathbb{E}_{x}\left[f\left(X_{t} / M_{t}\right)\right]=\pi^{-d / 2} \frac{\Gamma((d+\alpha) / 2)}{\Gamma(\alpha / 2)} \int_{\mathbb{S}^{d-1}} \sigma_{1}(\mathrm{~d} \phi) \int_{|w|<1} f(w) \frac{\left.\left.|1-| w\right|^{2}\right|^{\alpha / 2}}{|\phi-w|^{d}} \mathrm{~d} w,
$$

where $\sigma_{1}(\mathrm{~d} y)$ is the surface measure on $\mathbb{S}^{d-1}$, normalised to have unit mass.

Remark 1.6 Although we are dealing with the case $d \geq 2$, with the help of the duplication formula for gamma functions, we can verify that the above limiting identity agrees with the stationary distribution for the radially reflected process when $d=1$ given in Theorem 1.3 in [14] if we set $d=1$ and $\alpha \in(0,1)$.

We also note that the stationary distribution in the previous theorem is equal in law to the independent product of random variables $\mathrm{U} \times \sqrt{\mathrm{B}}$, where $\mathrm{U}$ is uniformly distributed on $\mathbb{S}^{d-1}$ and $\mathrm{B}$ is $\operatorname{arta}(d / 2, \alpha / 2)$ distribution. Indeed, suppose we take $f(w)=|w|^{2 \gamma} g(\arg (w))$ for $\gamma>0$, then we also see that

$$
\begin{aligned}
\lim _{t \rightarrow \infty} \mathbb{E}_{x}\left[f\left(X_{t} / M_{t}\right)\right]= & \frac{2 \Gamma((d+\alpha) / 2)}{\Gamma(d / 2) \Gamma(\alpha / 2)} \int_{\mathbb{S}^{d-1}} \sigma_{1}(\mathrm{~d} \phi) \int_{0}^{1} r^{2 \gamma+d-1} \\
& \left(1-r^{2}\right)^{\alpha / 2} \mathrm{~d} r \int_{\mathbb{S}^{d-1}} \frac{g(\theta)}{|\phi-r \theta|^{d}} \sigma_{1}(\mathrm{~d} \theta) .
\end{aligned}
$$


A Newton potential formula tells us that $\left(1-r^{2}\right) \int_{\mathbb{S}^{d-1}}|\phi-r \theta|^{-d} \sigma_{1}(\mathrm{~d} \phi)=1$, see for example Remark III.2.5 in [16], and hence, after an application of Fubini's theorem for the two spherical integrals and change of variable,

$$
\lim _{t \rightarrow \infty} \mathbb{E}_{x}\left[f\left(X_{t} / M_{t}\right)\right]=\frac{\Gamma((d+\alpha) / 2)}{\Gamma(d / 2) \Gamma(\alpha / 2)} \int_{0}^{1} u^{\gamma+\frac{d}{2}-1}(1-u)^{\frac{\alpha}{2}-1} \mathrm{~d} u \times \int_{\mathbb{S}^{d-1}} g(\theta) \sigma_{1}(\mathrm{~d} \theta),
$$

verifying the claimed distributional decomposition.

The remainder of this paper is structured as follows. In the next section we discuss the fundamental tool that allows us to conduct our analysis: an appropriate excursion theory of the underlying MAP $(\xi, \Theta)$. This may otherwise be understood as (up to a change of time and change of scale space) the excursion of $X$ from its radial minimum. With this in hand, we progress directly to the proof of Theorem 1.1 in Section 3. Thereafter, in Section 4, we introduce the so-called Riesz-Bogdan-Żak transform and discuss its relation to some of the key quantities that appear in the aforesaid radial fluctuation theory. Next, in Section 5 we analyse in more detail some specific identities pertaining to integration with respect to the excursion measure that appears in Section 2. These identities are then used to prove Theorem 1.3 in Section 6 and to prove the deep factorisation in Section 7. Finally, we deal with the stationary distribution, which is proved in Section 8.

\section{Radial Excursion Theory}

One of the principal tools that we will use in our computations is that of radial excursion theory of $X$ from its running minimum. In order to build such a theory, we return to the Lamperti-Kiu transformation (1.11). In the spirit of the discussion preceding Theorem 1.4, by considering, say, $\ell=\left(\ell_{t}, t \geq 0\right)$, the local time at 0 of the reflected Lévy process $\left(\xi_{t}-\underline{\xi}_{t}, t \geq 0\right)$, where $\underline{\xi}_{t}:=\inf _{s \leq t} \xi_{s}, t \geq 0$, we can build the descending ladder MAP $\left(\left(H_{t}^{-}, \Theta_{t}^{-}\right), t \geq 0\right)$, in the obvious way. As before, although the local time $\ell$ pertains to the reflected Lévy process $\xi-\xi$, we will see below that it serves as an adequate choice for the local time of the Markov process $(\xi-\xi, \Theta)$ on the set $\{0\} \times \mathbb{S}^{d-1}$ to the extent that we can use it in the context of Maisonneuve's exit formula.

More precisely, suppose we define $g_{t}=\sup \left\{s<t: \xi_{s}=\xi_{s}\right\}$, and recall that the regularity of $\xi$ for $(-\infty, 0)$ and $(0, \infty)$ ensures that it is well defined, as is $g_{\infty}=\lim _{t \rightarrow \infty} g_{t}$. Set

$$
\mathrm{d}_{t}=\inf \left\{s>t: \xi_{s}=\underline{\xi}_{s}\right\}
$$

and, for all $t>0$ such that $\mathrm{d}_{t}>\mathrm{g}_{t}$ the process

$$
\left(\epsilon_{\mathrm{g}_{t}}(s), \Theta_{\mathrm{g}_{t}}^{\epsilon}(s)\right):=\left(\xi_{\mathrm{g}_{t}+s}-\xi_{\mathrm{g}_{t}}, \Theta_{\mathrm{g}_{t}+s}\right), \quad s \leq \zeta_{\mathrm{g}_{t}}:=\mathrm{d}_{t}-\mathrm{g}_{t},
$$

codes the excursion of $(\xi-\xi, \Theta)$ from the set $\left(0, \mathbb{S}^{d-1}\right)$ which straddles time $t$. Such excursions live in the space $\mathbb{U}\left(\overline{\mathbb{R}} \times \mathbb{S}^{d-1}\right)$, the space of càdlàg paths

$$
\left(\epsilon, \Theta^{\epsilon}\right)=\left(\left(\epsilon(t), \Theta^{\epsilon}(t)\right): t \leq \zeta\right) \text { with lifetime } \zeta=\inf \{s>0: \epsilon(s)<0\}
$$

such that $\left(\epsilon(0), \Theta^{\epsilon}(0)\right) \in\{0\} \times \mathbb{S}^{d-1},\left(\epsilon(s), \Theta^{\epsilon}(s)\right) \in(0, \infty) \times \mathbb{S}^{d-1}$, for $0<s<\zeta$, and $\epsilon(\zeta) \in(-\infty, 0)$.

Taking account of the Lamperti-Kiu transform (1.11), it is natural to consider how the excursion of $(\xi-\underline{\xi}, \Theta)$ from $\{0\} \times \mathbb{S}^{d-1}$ translates into a radial excursion theory for the process

$$
Y_{t}:=\mathrm{e}^{\xi_{t}} \Theta_{t}, \quad t \geq 0 .
$$


Ignoring the time change in Eq. 1.11, we see that the radial minima of the process $Y$ agree with the radial minima of the stable process $X$. Indeed, an excursion of $(\xi-\underline{\xi}, \Theta)$ from $\{0\} \times \mathbb{S}^{d-1}$ constitutes an excursion of $\left(Y_{t} / \inf _{s \leq t}\left|Y_{s}\right|, t \geq 0\right)$, from $\mathbb{S}^{d-1}$, or equivalently an excursion of $Y$ from its running radial infimum. Moreover, we see that, for all $t>0$ such that $\mathrm{d}_{t}>\mathrm{g}_{t}$,

$$
Y_{g_{t}+s}=\mathrm{e}^{\xi_{g_{t}}} \mathrm{e}^{\epsilon_{g_{t}}(s)} \Theta_{g_{t}}^{\epsilon}(s)=\left|Y_{g_{t}}\right| \mathrm{e}^{\epsilon_{g_{t}}(s)} \Theta_{\mathrm{g}_{t}}^{\epsilon}(s), \quad s \leq \zeta_{g_{t}}
$$

This will be useful to keep in mind in the forthcoming excursion computations.

For $t>0$, let $R_{t}=\mathrm{d}_{t}-t$, and define the set $G=\left\{t>0: R_{t-}=0, R_{t}>0\right\}=\left\{g_{s}\right.$ : $s \geq 0\}$. The classical theory of exit systems in [17] (see Theorem (4.1) therein) now implies that there exists

an additive functional $\left(\Lambda_{t}, t \geq 0\right)$ and a family of excursion measures, $\left(\mathbb{N}_{\theta}, \theta \in \mathbb{S}^{d-1}\right)$

such that:

(i) $\Lambda$ is an additive functional of $(\xi, \Theta)$, has a bounded 1-potential and is carried by the set of times $\left\{t \geq 0:\left(\xi_{t}-\underline{\xi}_{t}, \Theta_{t}\right) \in\{0\} \times \mathbb{S}^{d-1}\right\}$,

(ii) the map $\theta \mapsto \mathbb{N}_{\theta}$ is an $\mathbb{S}^{d-1}$-indexed kernel on $\mathbb{U}\left(\mathbb{R} \times \mathbb{S}^{d-1}\right)$ such that $\mathbb{N}_{\theta}\left(1-e^{-\zeta}\right)<$ $\infty$ and $\mathbb{N}_{\theta}$, specifically, is carried by the set $\left\{\left(\epsilon(0+), \Theta^{\epsilon}(0)\right)=(0, \theta)\right\}$ and $\{\zeta>0\}$;

(iii) we have the exit formula

$$
\begin{gathered}
\mathbf{E}_{x, \theta}\left[\sum_{g \in G} F\left(\left(\xi_{s}, \Theta_{s}\right): s<g\right) H\left(\left(\epsilon_{g}, \Theta_{g}^{\epsilon}\right)\right)\right] \\
=\mathbf{E}_{x, \theta}\left[\int_{0}^{\infty} F\left(\left(\xi_{s}, \Theta_{s}\right): s<t\right) \mathbb{N}_{\Theta_{t}}\left(H\left(\epsilon, \Theta^{\epsilon}\right)\right) \mathrm{d} \Lambda_{t}\right],
\end{gathered}
$$

for $x \neq 0$, where $F$ is continuous on the space of càdlàg paths $\mathbb{D}\left(\mathbb{R} \times \mathbb{S}^{d-1}\right)$ and $H$ is measurable on the space of càdlàg paths $\mathbb{U}\left(\mathbb{R} \times \mathbb{S}^{d-1}\right)$;

(iv) under any measure $\mathbb{N}_{\theta}$ the process $\left(\left(\epsilon(s), \Theta^{\epsilon}(s)\right), s<\zeta\right)$ is Markovian with the same semigroup as $(\xi, \Theta)$ killed at its first hitting time of $(-\infty, 0] \times \mathbb{S}^{d-1}$.

The couple $(\Lambda, \mathbb{N}$.) is called an exit system. Note that in Maisonneuve's original formulation, the pair $\Lambda$ and the kernel $\mathbb{N}$ is not unique, but once $\Lambda$ is chosen the measures $\left(\mathbb{N}_{\theta}, \theta \in \mathbb{S}^{d-1}\right)$ are determined but for a $\Lambda$-neglectable set, i.e. a set $\mathcal{A}$ such that $\mathbf{E}_{x, \theta}\left(\int_{t \geq 0} 1_{\left\{\left(\xi_{s}-\xi_{s}, \Theta_{s}\right) \in \mathcal{A}\right\}} \mathrm{d} \Lambda_{s}\right)=0$.

Referring back to the first paragraph of this section, since $\ell$ is an additive functional with a bounded 1-potential, there is an exit system which corresponds to $(\ell, \mathbb{N}$.). Henceforth, this is the exit system we will work with and the system of excursion associated to it is what we call our radial excursion theory.

Before proceeding to make use of the exit system $(\ell, \mathbb{N}$.), let us make a further normalisation of $\ell$ (as a local time of the reflected Lévy process $\xi-\xi$, it is only defined up to a multiplicative constant). On account of the fact that $X$ is transient, in the sense of Eq. 1.3, we know that $\left(H^{-}, \Theta^{-}\right)$experiences killing at a rate that occurs, in principle, in a statedependent manner, specifically $\mathbb{N}_{\theta}(\zeta=\infty), \theta \in \mathbb{S}^{d-1}$. Isotropy allows us to conclude that all such rates take a common value and thanks to the arbitrary scaling of local time $\ell$, we can choose this common value to be unity. Said another way, $\ell_{\infty}$ is exponentially distributed with rate 1 . 
The importance of Eq. 2.1 can be seen, for example, when we consider the distribution of $X_{\mathrm{G}(\infty)}$. Indeed, we have for bounded measurable $f$ on $\mathbb{R}^{d}$,

$$
\begin{aligned}
\mathbb{E}_{x}\left[f\left(X_{\mathrm{G}(\infty)}\right)\right] & =\mathbf{E}_{\log |x|, \arg (x)}\left[\sum_{t \in G} f\left(\mathrm{e}^{\xi_{t}} \Theta_{t}\right) \mathbf{1}\left(\zeta_{t}=\infty\right)\right] \\
& =\mathbf{E}_{\log |x|, \arg (x)}\left[\int_{0}^{\infty} f\left(\mathrm{e}^{\xi_{t}} \Theta_{t}\right) \mathbb{N}_{\Theta_{t}}(\zeta=\infty) \mathrm{d} \ell_{t}\right] \\
& =\mathbf{E}_{\log |x|, \arg (x)}\left[\int_{0}^{\ell \infty} f\left(\mathrm{e}^{-H_{t}^{-}} \Theta_{t}^{-}\right) \mathbb{N}_{\Theta_{t}^{-}}(\zeta=\infty) \mathrm{d} t\right] \\
& =\int_{|z|<|x|} U_{x}^{-}(\mathrm{d} z) f(z) \mathbb{N}_{\arg (z)}(\zeta=\infty),
\end{aligned}
$$

where

$$
U_{x}^{-}(\mathrm{d} z):=\int_{0}^{\infty} \mathbf{P}_{\log |x|, \arg (x)}\left(\mathrm{e}^{-H_{t}^{-}} \Theta_{t}^{-} \in \mathrm{d} z, t<\ell_{\infty}\right) \mathrm{d} t, \quad|z| \leq|x|
$$

may be thought of as a potential. (It is worth noting here that the definition of $U_{x}^{-}$is designed specifically to look at the expected occupation measure of the radial minima in Cartesian coordinates, rather than in polar coordinates.)

In conclusion, we reach the identity

$$
\mathbb{E}_{x}\left[f\left(X_{\mathrm{G}(\infty)}\right)\right]=\int_{|z|<|x|} U_{x}^{-}(\mathrm{d} z) f(z)
$$

or equivalently, the law of $X_{\mathrm{G}(\infty)}$ under $\mathbb{P}_{x}, x \neq 0$, is nothing more than the measure $U_{x}^{-}(\mathrm{d} z),|z| \leq|x|$. From this analysis, in combination with Eq. 1.9, we also get another handy identity which will soon be of use. For $r<|x|, \mathbb{P}_{x}\left(\tau_{r}^{\oplus}=\infty\right)=\mathbb{P}_{x}\left(\left|X_{\mathrm{G}(\infty)}\right|>r\right)$ and hence, from Theorem 1.2 we have

$$
\int_{r<|z|<|x|} U_{x}^{-}(\mathrm{d} z)=\frac{\Gamma(d / 2)}{\Gamma((d-\alpha) / 2) \Gamma(\alpha / 2)} \int_{0}^{\left(|x|^{2} / r^{2}\right)-1}(u+1)^{-d / 2} u^{\alpha / 2-1} \mathrm{~d} u .
$$

Another identity where we gain some insight into the quantity $U_{x}^{-}$is the first passage result of Blumental-Getoor-Ray [3] which was already stated in Eq. 1.8. For example, the following identity emerges very quickly from Eq. 2.1. For bounded measurable functions $f, g$ on $\mathbb{R}^{d}$,

$$
\begin{aligned}
& \mathbb{E}_{x}\left[g\left(X_{\mathrm{G}\left(\tau_{1}^{\oplus}\right)}\right) f\left(X_{\tau_{1}^{\oplus}}\right) ; \tau_{1}^{\oplus}<\infty\right] \\
& =\int_{1<|z|<|x|} U_{x}^{-}(\mathrm{d} z) \int_{|y||z|<1} \mathbb{N}_{\arg (z)}\left(e^{\epsilon(\zeta)} \Theta^{\epsilon}(\zeta) \in \mathrm{d} y ; \zeta<\infty\right) g(z) f(|z| y) .
\end{aligned}
$$

With judicious computations in the spirit of those given above, there is scope to extract an identity for $U_{x}^{-}$in combination with Eq. 1.8. For example, suppose $v$ is the Lévy measure of the subordinator $H^{-}$. Developing (2.5), we can write

$$
\begin{aligned}
\mathbb{E}_{x}\left[f\left(\left|X_{\tau_{1}^{\oplus}}\right|\right) ; \tau_{1}^{\oplus}<\infty\right] & =\int_{1<|z|<|x|} U_{x}^{-}(\mathrm{d} z) \int_{y>\log |z|} \mathbb{N}_{\arg (z)}(|\epsilon(\zeta)| \in \mathrm{d} y ; \zeta<\infty) f\left(|z| \mathrm{e}^{-y}\right) \\
& =\int_{1<|z|<|x|} U_{x}^{-}(\mathrm{d} z) \int_{y>\log |z|} v(\mathrm{~d} y) f\left(|z| \mathrm{e}^{-y}\right)
\end{aligned}
$$


for $|x|>1$ and bounded measurable $f$ on $\mathbb{R}^{d}$, where we have appealed to isotropy to write $\mathbb{N}_{\arg (z)}(|\epsilon(\zeta)| \in \mathrm{d} y)=v(\mathrm{~d} y)$. On account of the fact that the Wiener-Hopf factorisation for $\xi$ is known, c.f. Eq. 1.6, the measure $v$ can written explicitly; see [5]. Indeed, the normalisation of $\ell$ is equivalent to the requirement that $\Phi^{-}(0)=1$, where $\Phi^{-}$is the Laplace exponent of $H^{-}$and hence

$$
\Phi^{-}(\lambda)=\int_{(0, \infty)}\left(1-\mathrm{e}^{-\lambda y}\right) v(\mathrm{~d} y)=\frac{\Gamma((d-\alpha) / 2) \Gamma((\lambda+d) / 2)}{\Gamma(d / 2) \Gamma((\lambda+d-\alpha) / 2)}, \quad \lambda \geq 0,
$$

which, inverting with the help of a change of variables and the beta integral (see also [5]), tells us that

$$
v(\mathrm{~d} y)=\frac{\alpha \Gamma((d-\alpha) / 2)}{\Gamma(d / 2) \Gamma(1-\alpha / 2)}\left(1-\mathrm{e}^{-2 y}\right)^{-\frac{\alpha}{2}-1} \mathrm{e}^{-d y} \mathrm{~d} y .
$$

Nonetheless, despite the fact that the left-hand side of Eqs. 2.6 and 2.7 are explicitly available, it seems here, and in other similar computations of this type, difficult to back out an expression for the measure $U_{x}^{-}$.

Whilst our approach will make use of some of the identities above, fundamentally we prove Theorem 1.1 via a method of approximation, out of which the expression we will obtain for $U_{x}^{-}$can be cleverly used, in conjunction of the excursion theory above, to derive a number of other identities.

\section{Proof of Theorem 1.1}

We start with some notation. First define, for $x \neq 0,|x|>r, \delta>0$ and continuous, positive and bounded $f$ on $\mathbb{R}^{d}$,

$$
\Delta_{r}^{\delta} f(x):=\frac{1}{\delta} \mathbb{E}_{x}\left[f\left(\arg \left(X_{\mathrm{G}_{\infty}}\right)\right),\left|X_{\mathrm{G}_{\infty}}\right| \in[r-\delta, r]\right] .
$$

The crux of our proof is to establish a limit of $\Delta_{r}^{\delta} f(x)$ in concrete terms as $\delta \rightarrow 0$.

Note that, by conditioning on first entry into the ball of radius $r$, we have, with the help of the first entrance law (1.8) and (2.3),

$$
\begin{aligned}
\Delta_{r}^{\delta} f(x) & =\frac{1}{\delta} \int_{|y| \in[r-\delta, r]} \mathbb{P}_{x}\left(X_{\tau_{r}^{\oplus}} \in \mathrm{d} y ; \tau_{r}^{\oplus}<\infty\right) \mathbb{E}_{y}\left[f\left(\arg \left(X_{\mathrm{G}_{\infty}}\right)\right) ;\left|X_{\mathrm{G}_{\infty}}\right| \in(r-\delta,|y|]\right] \\
& =\frac{1}{\delta} C_{\alpha, d} \int_{|y| \in[r-\delta, r]} \mathrm{d} y\left|\frac{r^{2}-|x|^{2}}{r^{2}-|y|^{2}}\right|^{\alpha / 2}|y-x|^{-d} \mathbb{E}_{y}\left[f\left(\arg \left(X_{\mathrm{G}_{\infty}}\right)\right) ;\left|X_{\mathrm{G}_{\infty}}\right| \in(r-\delta,|y|]\right] \\
& =\left.\left.\frac{1}{\delta} C_{\alpha, d}\left|r^{2}-\right| x\right|^{2}\right|^{\alpha / 2} \int_{|y| \in(r-\delta, r]} \mathrm{d} y \frac{|y-x|^{-d}}{\left.\left.\left|r^{2}-\right| y\right|^{2}\right|^{\alpha / 2}} \int_{r-\delta \leq|z| \leq|y|} U_{y}^{-}(\mathrm{d} z) f(\arg (z)), \quad \text { (3.1) }
\end{aligned}
$$

where we recall from Eq. 1.8 that $C_{\alpha, d}=\pi^{-(d / 2+1)} \Gamma(d / 2) \sin (\pi \alpha / 2)$. Our next objective is to try and replace $\int_{r-\delta \leq|z| \leq|y|} U_{y}^{-}(\mathrm{d} z) f(\arg (z))$ by a term of simpler form which can be asymptotically estimated in the limit as $\delta \rightarrow 0$. To this end, we need some technical lemmas.

Lemma 3.1 Suppose that $f$ is a bounded continuous function on $\mathbb{R}^{d}$ and $r>0$. Then

$$
\lim _{\delta \rightarrow 0} \sup _{|y| \in(r-\delta, r]}\left|\frac{\int_{r-\delta \leq|z| \leq|y|} U_{y}^{-}(\mathrm{d} z) f(z)}{\int_{r-\delta \leq|z| \leq|y|} U_{y}^{-}(\mathrm{d} z)}-f(y)\right|=0 .
$$


Proof Suppose that $\mathcal{C}_{r, \delta, \varepsilon}(y)$ is the geometric region which coincides with the intersection of a cone with axis along $y$ with radial extent $2 \varepsilon$, say $\mathcal{C}_{\varepsilon}$, and the annulus $\left\{z \in \mathbb{R}^{d}: r-\delta \leq\right.$ $|z| \leq r\}$; see Fig. 1. Chose $\varepsilon, \delta$ such that

$$
\sup _{z \in \mathcal{C}_{r, \delta, \varepsilon}(y)}|f(z)-f(y)|<\varepsilon^{\prime},
$$

for some choice of $\varepsilon^{\prime} \ll 1$.

We have

$$
\begin{aligned}
& \sup _{|y| \in(r-\delta, r]}\left|\frac{\int_{r-\delta \leq|z| \leq|y|} U_{y}^{-}(\mathrm{d} z) f(z)}{\int_{r-\delta \leq|z| \leq|y|} U_{y}^{-}(\mathrm{d} z)}-f(y)\right| \\
& \leq \varepsilon^{\prime}+2|| f \|_{\infty} \sup _{|y| \in(r-\delta, r]} \frac{\int_{r-\delta \leq|z| \leq|y|} U_{y}^{-}(\mathrm{d} z) \mathbf{1}\left(z \notin \mathcal{C}_{r, \delta, \varepsilon}(y)\right)}{\int_{r-\delta \leq|z| \leq|y|} U_{y}^{-}(\mathrm{d} z)} .
\end{aligned}
$$

In order to deal with the second term in the right-hand side above, taking the example computations of Eqs. 2.5 and 2.6, note that, for $|y| \in(r-\delta, r]$,

$$
\begin{aligned}
\sup _{|y| \in(r-\delta, r]} \int_{r-\delta \leq|z| \leq|y|} & U_{y}^{-}(\mathrm{d} z) \mathbf{1}\left(z \notin \mathcal{C}_{r, \delta, \varepsilon}(y)\right) v\left(\log \left(\frac{|z|}{r-\delta}\right), \infty\right) \\
= & \sup _{|y| \in(r-\delta, r]} \mathbb{P}_{y}\left(X_{\tau_{r-\delta}^{\oplus}-} \notin \mathcal{C}_{r, \delta, \varepsilon}(y), \tau_{r-\delta}^{\oplus}<\infty\right) \\
= & \sup _{\beta \in(r-\delta, r]} \mathbb{P}_{\beta 1}\left(X_{\tau_{r-\delta}^{\oplus}} \notin \mathcal{C}_{r, \delta, \varepsilon}(\beta 1), \tau_{r-\delta}^{\oplus}<\infty\right) \\
\leq & \sup _{\beta \in(r-\delta, r]} \mathbb{P}_{\beta 1}\left(\Theta_{\sigma_{r-\delta}^{-}}^{-} \notin \mathcal{C}_{\varepsilon} \cap \mathbb{S}^{d-1}, \sigma_{r-\delta}^{-}<\infty\right) \\
\leq & \sup _{\beta \in(r-\delta, r]} \mathbb{P}_{\beta 1}\left(v_{\varepsilon}<\sigma_{r-\delta}^{-}\right) \\
\leq & \mathbb{P}_{r 1}\left(v_{\varepsilon}<\sigma_{r-\delta}^{-}\right)
\end{aligned}
$$

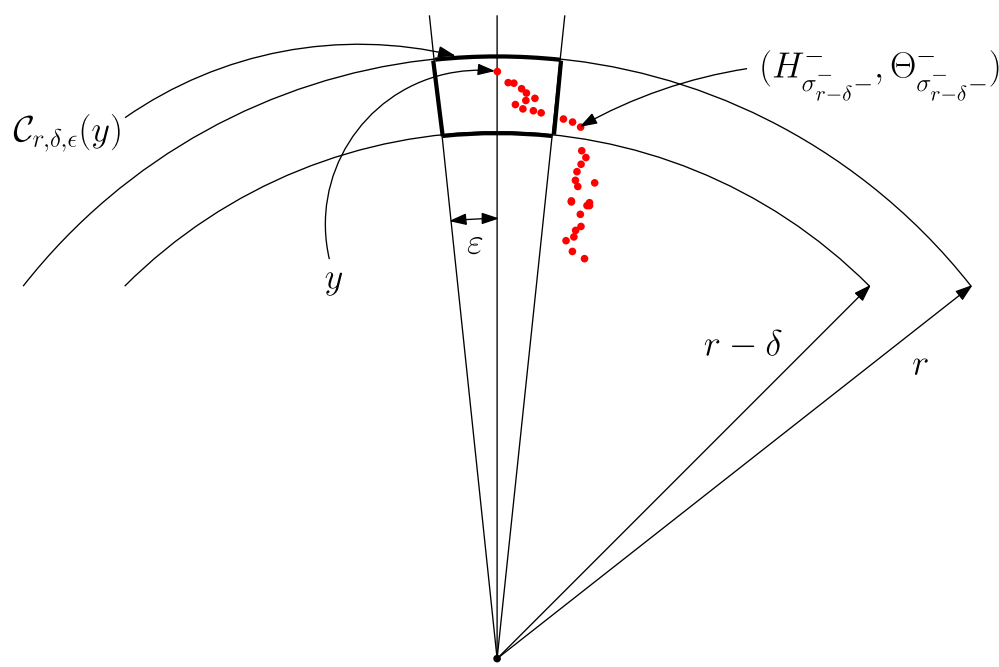

Fig. 1 The process $\left(H^{-}, \Theta^{-}\right)$in relation to the domain $\mathcal{C}_{r, \delta, \varepsilon}(y)$ 
where $1=(1,0, \cdots, 0)$ is the 'North Pole' on $\mathbb{S}^{d-1}, \sigma_{r-\delta}^{-}=\inf \left\{t>0: H_{t}^{-}<r-\delta\right\}$ and $v_{\varepsilon}=\inf \left\{t>0: \Theta_{t}^{-} \notin \mathcal{C}_{\varepsilon} \cap \mathbb{S}^{d-1}\right\}$. Right-continuity of paths now ensures that the right-hand side above tends to zero as $\delta \rightarrow 0$.

On the other hand, from Eq. 2.4

$$
\int_{r-\delta \leq|z| \leq|y|} U_{y}^{-}(\mathrm{d} z)=\mathbb{P}_{y}\left(\tau_{r-\delta}^{\oplus}=\infty\right)=\mathbb{P}_{\frac{|y|}{(r-\delta)} 1}\left(\tau_{1}^{\oplus}=\infty\right),
$$

where we have used isotropy in the final equality and from Eqs. 1.9 and 2.7 a rather elementary computation shows that

$$
\lim _{\eta \downarrow 1} v(\log \eta, \infty) \mathbb{P}_{\eta 1}\left(\tau_{1}^{\oplus}=\infty\right)=\frac{1}{\Gamma(1+\alpha / 2) \Gamma(1-\alpha / 2)} .
$$

Hence

$$
\begin{aligned}
& \lim _{\delta \rightarrow 0} \sup _{|y| \in(r-\delta, r]} \frac{\int_{r-\delta \leq|z| \leq|y|} U_{y}^{-}(\mathrm{d} z) \mathbf{1}\left(z \notin \mathcal{C}_{r, \delta, \varepsilon}(y)\right)}{\int_{r-\delta \leq|z| \leq|y|} U_{y}^{-}(\mathrm{d} z)} \\
& \leq \lim _{\delta \rightarrow 0} \sup _{|y| \in(r-\delta, r]} \frac{\int_{r-\delta \leq|z| \leq|y|} U_{y}^{-}(\mathrm{d} z) \mathbf{1}\left(z \notin \mathcal{C}_{r, \delta, \varepsilon}(y)\right) \frac{v(\log (|z| /(r-\delta)), \infty)}{v(\log (|z| /(r-\delta)), \infty)}}{\int_{r-\delta \leq|z| \leq|y|} U_{y}^{-}(\mathrm{d} z)} \\
& \leq \lim _{\delta \rightarrow 0} \sup _{|y| \in(r-\delta, r]} \frac{\int_{r-\delta \leq|z| \leq|y|} U_{y}^{-}(\mathrm{d} z) \mathbf{1}\left(z \notin \mathcal{C}_{r, \delta, \varepsilon}(y)\right) v(\log (|z| /(r-\delta)), \infty)}{v(\log (|y| /(r-\delta)), \infty) \mathbb{P}_{y}\left(\tau_{r-\delta}^{\oplus}=\infty\right)} \\
& \leq \lim _{\delta \rightarrow 0} \sup _{1<\eta<1+\frac{\delta}{(r-\delta)}} \frac{\mathbb{P}_{r 1}\left(v_{\varepsilon}<\sigma_{r-\delta}^{-}\right)}{v(\log \eta, \infty) \mathbb{P}_{\eta 1}\left(\tau_{1}^{\oplus}=\infty\right)} \\
& =0
\end{aligned}
$$

and thus plugging this back into Eq. 3.2 gives the result.

With Lemma 3.1 in hand, noting in particular the representation (3.4), we can now return to Eq. 3.1 and note that, for each $\varepsilon>0$, we can choose $\delta$ sufficiently small such that

$$
\Delta_{r}^{\delta} f(x)=D(\varepsilon) \Delta_{r}^{\delta} 1(x)+\left.\left.\frac{1}{\delta} C_{\alpha, d}\left|r^{2}-\right| x\right|^{2}\right|^{\alpha / 2} \int_{|y| \in(r-\delta, r]} \mathrm{d} y \frac{|y-x|^{-d}}{\left.\left.\left|r^{2}-\right| y\right|^{2}\right|^{\alpha / 2}} f(\arg (y)) \mathbb{P}_{y}\left(\tau_{r-\delta}^{\oplus}=\infty\right),
$$

where, $|D(\varepsilon)|<\varepsilon$ and for $|x|>r$,

$$
\begin{aligned}
\limsup _{\delta \rightarrow 0}\left|\Delta_{r}^{\delta} 1(x)\right| & \leq \limsup _{\delta \rightarrow 0}\left|\frac{1}{\delta} C_{\alpha, d}\right| r^{2}-\left.|x|^{2}\right|^{\alpha / 2} \int_{|y| \in(r-\delta, r]} \mathrm{d} y \frac{|y-x|^{-d}}{\left.\left.\left|r^{2}-\right| y\right|^{2}\right|^{\alpha / 2}} \mathbb{P}_{y}\left(\tau_{r-\delta}^{\oplus}=\infty\right) \mid \\
& =\limsup _{\delta \rightarrow 0}\left|\frac{1}{\delta}\left(\mathbb{P}_{x}\left(\tau_{r-\delta}^{\oplus}=\infty\right)-\mathbb{P}_{x}\left(\tau_{r}^{\oplus}=\infty\right)\right)\right| \\
& =\frac{\Gamma(d / 2)}{\Gamma((d-\alpha) / 2) \Gamma(\alpha / 2)}\left|\frac{\mathrm{d}}{\mathrm{d} v} \int_{0}^{\left(|x|^{2} / v^{2}\right)-1}(u+1)^{-d / 2} u^{\alpha / 2-1} \mathrm{~d} u\right|_{v=r} \\
& =\frac{2 \Gamma(d / 2)}{\Gamma((d-\alpha) / 2) \Gamma(\alpha / 2)}\left(|x|^{2}-r^{2}\right)^{\alpha / 2-1} r^{d-1-\alpha}|x|^{2-d}
\end{aligned}
$$

where in the third equality we have used Eq. 1.9. 
We can now say that, if the limit exists,

$$
\begin{aligned}
& \lim _{\delta \rightarrow 0} \Delta_{r}^{\delta} f(x) \\
= & \left.\left.\lim _{\delta \rightarrow 0} C_{\alpha, d}\left|r^{2}-\right| x\right|^{2}\right|^{\alpha / 2} \frac{1}{\delta} \int_{|y| \in(r-\delta, r]} \mathrm{d} y \frac{|y-x|^{-d}}{\left.\left.\left|r^{2}-\right| y\right|^{2}\right|^{\alpha / 2}} f(\arg (y)) \mathbb{P}_{y}\left(\tau_{r-\delta}^{\oplus}=\infty\right) \\
= & \left.\left.\lim _{\delta \rightarrow 0} C_{\alpha, d}\left|r^{2}-\right| x\right|^{2}\right|^{\alpha / 2} \frac{1}{\delta} \int_{r-\delta}^{r} \rho^{d-1} \mathrm{~d} \rho \int_{\rho \mathbb{S}^{d-1}} \sigma_{\rho}(\mathrm{d} \theta) \frac{|\rho \theta-x|^{-d}}{\left|r^{2}-\rho^{2}\right|^{\alpha / 2}} f(\theta) \mathbb{P}_{\rho \theta}\left(\tau_{r-\delta}^{\oplus}=\infty\right) \\
= & \left.\left.\lim _{\delta \rightarrow 0} C_{\alpha, d}\left|r^{2}-\right| x\right|^{2}\right|^{\alpha / 2} \frac{1}{\delta} \int_{r-\delta}^{r} \rho^{d-1} \mathrm{~d} \rho \frac{\mathbb{P}_{\rho 1}\left(\tau_{r-\delta}^{\oplus}=\infty\right)}{\left|r^{2}-\rho^{2}\right|^{\alpha / 2}} \int_{\rho \mathbb{S}^{d-1}} \sigma_{\rho}(\mathrm{d} \theta)|\rho \theta-x|^{-d} f(\theta),
\end{aligned}
$$

where, in the second equality, we have switched from $d$-dimensional Lebesgue measure to the generalised polar coordinate measure $\rho^{d-1} \mathrm{~d} \rho \times \sigma_{\rho}(\mathrm{d} \theta)$, so that $\rho>0$ is the radial distance from the origin and $\sigma_{\rho}(\mathrm{d} \theta)$ is the surface measure on $\rho \mathbb{S}^{d-1}$, normalised to have unit mass. In the third equality we have used isotropy to write $\mathbb{P}_{\rho \theta}\left(\tau_{r-\delta}^{\oplus}=\infty\right)=\mathbb{P}_{\rho 1}\left(\tau_{r-\delta}^{\oplus}=\right.$ $\infty)$ for $\theta \in \mathbb{S}^{d-1}$.

Noting the continuity of the integral $\int_{\rho \mathbb{S}^{d-1}} \sigma_{\rho}(\mathrm{d} \theta)|\rho 1-x|^{-d} f(\theta)$ in $\rho$, the proof of Theorem 1.1 is complete as soon as we can evaluate

$$
\lim _{\delta \rightarrow 0} \frac{1}{\delta} \int_{r-\delta}^{r} \rho^{d-1} \mathrm{~d} \rho \frac{\mathbb{P}_{\rho 1}\left(\tau_{r-\delta}^{\oplus}=\infty\right)}{\left|r^{2}-\rho^{2}\right|^{-\alpha / 2}} .
$$

To this end, we need a technical lemma.

Lemma 3.2 Let $D_{\alpha, d}=\Gamma(d / 2) / \Gamma((d-\alpha) / 2) \Gamma(\alpha / 2)$. Then

$$
\lim _{\delta \rightarrow 0} \sup _{\rho \in[r-\delta, r]}\left|\left(\rho^{2}-(r-\delta)^{2}\right)^{-\alpha / 2} r^{\alpha} \mathbb{P}_{\rho 1}\left(\tau_{r-\delta}^{\oplus}=\infty\right)-\frac{2 D_{\alpha, d}}{\alpha}\right|=0
$$

Proof Appealing to Eq. 1.9, we start by noting that

$$
\begin{aligned}
& \sup _{\rho \in[r-\delta, r]}\left|D_{\alpha, d} \int_{0}^{\rho^{2} /(r-\delta)^{2}-1} u^{\alpha / 2-1} \mathrm{~d} u-\mathbb{P}_{\rho 1}\left(\tau_{r-\delta}^{\oplus}=\infty\right)\right| \\
\leq & \sup _{\rho \in[r-\delta, r]} D_{\alpha, d} \int_{0}^{\rho^{2} /(r-\delta)^{2}-1}\left|(1+u)^{-d / 2}-1\right| u^{\alpha / 2-1} \mathrm{~d} u \\
\leq & D_{\alpha, d}\left|1-\frac{(r-\delta)^{d}}{r^{d}}\right| \frac{2}{\alpha}\left(r^{2}-(r-\delta)^{2}\right)^{\alpha / 2}(r-\delta)^{-\alpha},
\end{aligned}
$$

which tends to zero as $\delta \rightarrow 0$. Furthermore,

$$
\begin{aligned}
& \sup _{\rho \in[r-\delta, r]}\left|D_{\alpha, d} \int_{0}^{\rho^{2} /(r-\delta)^{2}-1} u^{\alpha / 2-1} \mathrm{~d} u-\frac{2 D_{\alpha, d}}{\alpha}\left(\rho^{2}-(r-\delta)\right)^{\alpha / 2} r^{-\alpha}\right| \\
= & \sup _{\rho \in[r-\delta, r]} \frac{2 D_{\alpha, d}}{\alpha}\left(\rho^{2}-(r-\delta)^{2}\right)^{\alpha / 2}\left|(r-\delta)^{-\alpha}-r^{-\alpha}\right| \\
\leq & \frac{2 D_{\alpha, d}}{\alpha}\left(r^{2}-(r-\delta)\right)^{\alpha / 2}\left|(r-\delta)^{-\alpha}-r^{-\alpha}\right|,
\end{aligned}
$$


which also tends to zero as $\delta \rightarrow 0$. Summing (3.7) and (3.8) in the context of the triangle inequality and dividing by $r^{-\alpha}\left(r^{2}-(r-\delta)\right)^{\alpha / 2}$ we can also deduce that

$$
\lim _{\delta \rightarrow 0} \sup _{\rho \in[r-\delta, r]}\left|\left(\rho^{2}-(r-\delta)^{2}\right)^{-\alpha / 2} r^{\alpha} \mathbb{P}_{\rho 1}\left(\tau_{r-\delta}^{\oplus}=\infty\right)-\frac{2 D_{\alpha, d}}{\alpha}\right|=0,
$$

and the lemma is proved.

We are now ready to prove (3.6), and identify its limit, thereby completing the proof of Theorem 1.1. Appealing to Lemma 3.2, for all $\varepsilon>0$, there exists a $\delta$ sufficiently small,

$$
\begin{aligned}
& \left|\frac{1}{\delta} \int_{r-\delta}^{r} \mathrm{~d} \rho \frac{\mathbb{P}_{\rho 1}\left(\tau_{r-\delta}^{\oplus}=\infty\right)}{\left(r^{2}-\rho^{2}\right)^{\alpha / 2}}-\frac{2 D_{\alpha, d} r^{-\alpha}}{\alpha} \frac{1}{\delta} \int_{r-\delta}^{r} \mathrm{~d} \rho \frac{\left(\rho^{2}-(r-\delta)^{2}\right)^{\alpha / 2}}{\left(r^{2}-\rho^{2}\right)^{\alpha / 2}}\right| \\
& <\frac{\varepsilon}{\delta} \int_{r-\delta}^{r} \mathrm{~d} \rho \frac{\left(\rho^{2}-(r-\delta)^{2}\right)^{\alpha / 2}}{\left(r^{2}-\rho^{2}\right)^{\alpha / 2}} .
\end{aligned}
$$

Next note that, with the help of he substitution $\rho=(r-\delta)+u \delta$, it is straightforward to show that

$$
\lim _{\delta \rightarrow 0} \frac{1}{\delta} \int_{r-\delta}^{r} \mathrm{~d} \rho \frac{\left(\rho^{2}-(r-\delta)^{2}\right)^{\alpha / 2}}{\left(r^{2}-\rho^{2}\right)^{\alpha / 2}}=\Gamma(1-\alpha / 2) \Gamma(1+\alpha / 2) .
$$

Putting the pieces together, we can take limits in Eqs. 3.9, using 3.10, to deduce that

$$
\lim _{\delta \rightarrow 0} \frac{1}{\delta} \int_{r-\delta}^{r} \mathrm{~d} \rho \frac{\mathbb{P}_{\rho 1}\left(\tau_{r-\delta}^{\oplus}=\infty\right)}{\left(r^{2}-\rho^{2}\right)^{\alpha / 2}}=\frac{2}{\alpha} D_{\alpha, d} \Gamma(1-\alpha / 2) \Gamma(1+\alpha / 2) r^{-\alpha}
$$

which, in turn, can be plugged into Eq. 3.5 and we find that

$$
\begin{aligned}
\lim _{\delta \rightarrow 0} \Delta_{r}^{\delta} f(x) & =\left.\left.\frac{2}{\alpha} D_{\alpha, d} \Gamma(1-\alpha / 2) \Gamma(1+\alpha / 2) C_{\alpha, d} r^{d-\alpha-1}\left|r^{2}-\right| x\right|^{2}\right|^{\alpha / 2} \int_{r \mathbb{S}^{d-1}} \sigma_{\rho}(\mathrm{d} \theta)|r \theta-x|^{-d} f(\theta) \\
& =\left.\left.\pi^{-d / 2} \frac{\Gamma(d / 2)^{2}}{\Gamma((d-\alpha) / 2) \Gamma(\alpha / 2)} r^{d-\alpha-1}\left|r^{2}-\right| x\right|^{2}\right|^{\alpha / 2} \int_{r \mathbb{S}^{d-1}} \sigma_{\rho}(\mathrm{d} \theta)|r \theta-x|^{-d} f(\theta) .
\end{aligned}
$$

Now suppose that $g$ is another bounded measurable function on $[0, \infty)$, then

$$
\begin{aligned}
& \mathbb{E}_{x}\left[g\left(\left|X_{\mathrm{G}(\infty)}\right|\right) f\left(\arg \left(X_{\mathrm{G}(\infty)}\right)\right)\right] \\
= & \pi^{-d / 2} \frac{\Gamma(d / 2)^{2}}{\Gamma((d-\alpha) / 2) \Gamma(\alpha / 2)} \int_{0}^{|x|} \int_{r \mathbb{S}^{d-1}} r^{d-1} \mathrm{~d} r \sigma_{\rho}(\mathrm{d} \theta) \frac{\left.\left.\left|r^{2}-\right| x\right|^{2}\right|^{\alpha / 2}}{r^{\alpha}|r \theta-x|^{d}} f(\theta) g(r) \\
= & \pi^{-d / 2} \frac{\Gamma(d / 2)^{2}}{\Gamma((d-\alpha) / 2) \Gamma(\alpha / 2)} \int_{|y|<|x|} \frac{\left.|| y\right|^{2}-\left.|x|^{2}\right|^{\alpha / 2}}{|y|^{\alpha}|y-x|^{d}} f(\arg (y)) g(|y|) \mathrm{d} y,
\end{aligned}
$$

which is equivalent to the statement of Theorem 1.1.

\section{Riesz-Bogdan-Żak Transform and MAP Duality}

Recently, Bogdan and Żak [4] used an idea of Riesz from classical potential analysis to understand the relationship between a stable process and its transformation through a simple sphere inversion. (See also Alili et al. [1] and Kyprianou [11]). Suppose we write $K x=x /|x|^{2}, x \in \mathbb{R}^{d}$ for the classical inversion of space through the sphere $\mathbb{S}^{d-1}$. Then in 
dimension $d \geq 2$, Bogdan and Żak [4] prove that, for $x \neq 0,\left(K X_{\eta(t)}, t \geq 0\right)$ under $\mathbb{P}_{K x}$ is equal in law to $\left(X_{t}, t \geq 0\right)$ under $\mathbb{P}_{x}^{\circ}$, where

$$
\left.\frac{\mathrm{d} \mathbb{P}_{x}^{\circ}}{\mathrm{d} \mathbb{P}_{x}}\right|_{\sigma\left(X_{s}: s \leq t\right)}=\frac{\left|X_{t}\right|^{\alpha-d}}{|x|^{\alpha-d}}, \quad t \geq 0
$$

and $\eta(t)=\inf \left\{s>0: \int_{0}^{s}\left|X_{u}\right|^{-2 \alpha} \mathrm{d} u>t\right\}$. It was shown in Kyprianou et al. [15] that $\mathbb{P}_{x}^{\circ}$, $x \in \mathbb{R}^{d} \backslash\{0\}$ can be understood, in the appropriate sense, as the stable process conditioned to be continuously absorbed at the origin. Indeed, as far as the underlying $\operatorname{MAP}(\xi, \Theta)$ is concerned, we see that $-\mathrm{i}(\alpha-d)$ is a root of the exponent (1.6) and the change of measure (4.1) corresponds to an Esscher transform of the Lévy process $\xi$, rendering it a process which drifts to $-\infty$. Thus, an application of the optimal stopping theorem shows that Eq. 4.1 is equivalent to the change of measure for $\xi$

$$
\left.\frac{\mathrm{d} \mathbf{P}_{x, \theta}^{\circ}}{\mathrm{d} \mathbf{P}_{x, \theta}}\right|_{\sigma\left(\left(\xi_{s}, \Theta_{s}\right): s \leq t\right)}=\mathrm{e}^{(\alpha-d)\left(\xi_{t}-x\right)}, \quad t \geq 0
$$

Following the reasoning in the $d$-dimensional case of Theorem 3.7 of [1] and the 1dimensional case in [11], it is not difficult to show that the space-time transformed process $\left(K X_{\eta(t)}, t \geq 0\right)$ is the Lamperti-Kiu transform of the MAP $(-\xi, \Theta)$. Therefore, at the level of MAPs, the Riesz-Bogdan-Żak transform says that $(\xi, \Theta)$ under the change of measure (4.2), when issued from $(\log |x|, \arg (x)), x \in \mathbb{R}$, is equal in law to $(-\xi, \Theta)$ when issued from $(-\log |x|, \arg (x))$.

An interesting consequence of this is that the Riesz-Bogdan-Żak transform provides an efficient way to analyse radial ascending properties of $X$, where previously we have studied its descending properties. That is to say, it offers the opportunity to study aspects of the process $\left(H^{+}, \Theta^{+}\right)$. A good case in point in this respect is the analogue of the potential $U_{x}^{-}(\mathrm{d} y),|y|<|x|$.

For convenience, note from Theorem 1.1 and Eq. 2.3 that establishing the law of $X_{\mathrm{G}(\infty)}$ is equivalent to obtaining an explicit identity for $U_{x}^{-}(\mathrm{d} y),|y|<|x|$ and this we have already done. Specifically, for all $|x|>0$,

$$
U_{x}^{-}(\mathrm{d} y)=\pi^{-d / 2} \frac{\Gamma(d / 2)^{2}}{\Gamma((d-\alpha) / 2) \Gamma(\alpha / 2)} \frac{\left.|| y\right|^{2}-\left.|x|^{2}\right|^{\alpha / 2}}{|y|^{\alpha}|y-x|^{d}}, \quad|y|<|x| .
$$

On the other hand, recalling that $\lim _{t \rightarrow \infty}\left|X_{t}\right|=\infty$, which implies that $\lim _{t \rightarrow \infty} \xi_{t}=\infty$ and hence $L_{\infty}=\infty$, we define

$$
U_{x}^{+}(\mathrm{d} z)=\int_{0}^{\infty} \mathbf{P}_{\log |x|, \arg (x)}\left(\mathrm{e}^{H_{t}^{+}} \Theta_{t}^{+} \in \mathrm{d} z\right) \mathrm{d} t, \quad|z| \geq|x| .
$$

Then the Riesz-Bogdan-Żak transform ensures that, for Borel $A \subseteq\left\{z \in \mathbb{R}^{d}:|z|<|x|\right\}$,

$$
\frac{|z|^{\alpha-d}}{|x|^{\alpha-d}} \mathbf{P}_{\log |x|, \arg (x)}\left(\mathrm{e}^{H_{t}^{+}} \Theta_{t}^{+} \in A\right)=\mathbf{P}_{-\log |x|, \arg (x)}\left(\mathrm{e}^{-H_{t}^{-}} \Theta_{t}^{-} \in K A, t<\ell_{\infty}\right)
$$

where $K A=\{K z: z \in A\}$. Hence, for $|x|>0$,

$$
\begin{aligned}
U_{x}^{+}(\mathrm{d} z) & =\pi^{-d / 2} \frac{\Gamma(d / 2)^{2}}{\Gamma((d-\alpha) / 2) \Gamma(\alpha / 2)} \frac{\left.|| z\right|^{-2}-\left.|x|^{-2}\right|^{\alpha / 2}}{|z|^{-\alpha}\left|\left(z /|z|^{2}\right)-\left(x /|x|^{2}\right)\right|^{d}} \frac{|x|^{\alpha-d}}{|z|^{\alpha-d}} \frac{\mathrm{d} z}{|z|^{2 d}} \\
& =\pi^{-d / 2} \frac{\Gamma(d / 2)^{2}}{\Gamma((d-\alpha) / 2) \Gamma(\alpha / 2)} \frac{\left.|| z\right|^{2}-\left.|x|^{2}\right|^{\alpha / 2}}{|z|^{\alpha}|x-z|^{d}} \mathrm{~d} z, \quad|z|>|x| .
\end{aligned}
$$


where we have used the fact that $\mathrm{d} y=|z|^{-2 d} \mathrm{~d} z$, when $y=K z$, and

$$
|K x-K z|=\frac{|x-z|}{|x||z|} .
$$

One notices that the identities for the potential measures $U_{x}^{-}(\mathrm{d} z)$ and $U_{x}^{+}(\mathrm{d} z)$ are identical albeit that the former is supported on $|z|<|x|$ and the latter on $|z|>|x|$. This can be explained e.g. via the Riesz-Bogdan-Żak transformation. These expressions will be of use to us in due course.

\section{Integration with Respect to the Excursion Measure}

In order to proceed with some of the other fluctuation identities and the deep factorisation, we need to devote some time to compute in explicit detail the excursion occupation functionals

$$
\mathbb{N}_{\theta}\left(\int_{0}^{\zeta} g\left(\mathrm{e}^{\epsilon}(s) \Theta^{\epsilon}(s)\right) \mathrm{d} s\right), \quad \theta \in \mathbb{S}^{d-1},
$$

and the excursion overshoot

$$
\mathbb{N}_{\theta}\left(f\left(\mathrm{e}^{\epsilon(\zeta)} \Theta^{\epsilon}(\zeta)\right) ; \zeta<\infty\right), \quad \theta \in \mathbb{S}^{d-1},
$$

for non-negative, measurable $f$ and $g$.

The way we do this is to use Lemma 3.1 to scale out the quantity of interest from a fluctuation identity in which it is placed together with the potential $U_{x}^{-}$. Let us start with the excursion overshoot in Eq. 5.2.

Proposition 5.1 For $\theta \in \mathbb{S}^{d-1}$, we have

$$
\begin{aligned}
& \mathbb{N}_{\theta}\left(\mathrm{e}^{\epsilon(\zeta)} \Theta^{\epsilon}(\zeta) \in \mathrm{d} y ; \zeta<\infty\right) \\
= & \left.\left.\frac{\alpha \pi^{-d / 2}}{2} \frac{\Gamma((d-\alpha) / 2)}{\Gamma(1-\alpha / 2)}|1-| y\right|^{2}\right|^{-\alpha / 2}|\theta-y|^{-d} \mathrm{~d} y, \quad|y| \leq 1 .
\end{aligned}
$$

Proof Take $|x|>r>r_{0}>0$ and suppose that $f: \mathbb{R}^{d} \mapsto[0, \infty)$ is continuous with support which is compactly embedded in the ball of radius $r_{0}$. We have, on the one hand, from Eq. 1.8, the identity

$\mathbb{E}_{x}\left[f\left(X_{\tau_{r}^{\oplus}}\right) ; \tau_{r}^{\oplus}<\infty\right]=\pi^{-(d / 2+1)} \Gamma(d / 2) \sin \left(\frac{\pi \alpha}{2}\right) \int_{|y|<r} \frac{\left.\left.\left|r^{2}-\right| x\right|^{2}\right|^{\alpha / 2}}{\left.\left.\left|r^{2}-\right| y\right|^{2}\right|^{\alpha / 2}}|x-y|^{-d} f(y) \mathrm{d} y$.

On the other hand, from Eq. 2.5, we also have

$$
\mathbb{E}_{x}\left[f\left(X_{\tau_{r}^{\oplus}}\right) ; \tau_{r}^{\oplus}<\infty\right]=\int_{r<|z|<|x|} U_{x}^{-}(\mathrm{d} z) \int_{|y| z \mid<r} \mathbb{N}_{\arg (z)}\left(f\left(|z| e^{\epsilon(\zeta)} \Theta^{\epsilon}(\zeta)\right) ; \zeta<\infty\right) .
$$

Note that, for each $z \in \mathbb{R}^{d} \backslash\{0\}$,

$$
z \mapsto \mathbb{N}_{\arg (z)}\left(f\left(|z| e^{\epsilon(\zeta)} \Theta^{\epsilon}(\zeta)\right) ; \zeta<\infty\right)
$$

is bounded thanks to the fact that $f$ is bounded and its support is compactly embedded in the unit ball of radius $r_{0}$. Indeed, there exists an $\varepsilon>0$, which depends only on the support of $f$, such that

$$
\sup _{r<|z|<|x|}\left|\mathbb{N}_{\arg (z)}\left(f\left(|z| e^{\epsilon(\zeta)} \Theta^{\epsilon}(\zeta)\right) ; \zeta<\infty\right)\right| \leq\|f\|_{\infty} v\left(-\log \left(\left(r_{0}-\varepsilon\right), \infty\right)<\infty .\right.
$$


For any $a \in \mathbb{S}^{d-1}$, the operation $\star a$ rotates the sphere so that the 'North Pole', $1=$ $(1,0, \cdots, 0) \in \mathbb{S}^{d-1}$ moves to $a$. Write can thus write.

$$
\mathbb{N}_{\arg (z)}\left(f\left(|z| e^{\epsilon(\zeta)} \Theta^{\epsilon}(\zeta)\right) ; \zeta<\infty\right)=\mathbb{N}_{1}\left(f\left(|z| e^{\epsilon(\zeta)} \Theta^{\epsilon}(\zeta) \star \arg (z)\right) ; \zeta<\infty\right) .
$$

Using a straightforward dominated convergence argument, we see that $\mathbb{N}_{\arg (z)}\left(f\left(|z| e^{\epsilon(\zeta)} \Theta^{\epsilon}(\zeta)\right) ; \zeta<\infty\right)$ is continuous in $z$ thanks to the continuity of $f$.

Appealing to Lemma 3.1, we thus have that

$$
\begin{aligned}
& \mathbb{N}_{\arg (x)}\left(f\left(|x| e^{\epsilon(\zeta)} \Theta^{\epsilon}(\zeta)\right) ; \zeta<\infty\right) \\
= & \lim _{r \uparrow|x|} \frac{\int_{r<|z|<|x|} U_{x}^{-}(\mathrm{d} z) \int_{|y||z|<r} \mathbb{N}_{\arg (z)}\left(f\left(|z| e^{\epsilon(\zeta)} \Theta^{\epsilon}(\zeta)\right) ; \zeta<\infty\right)}{\int_{r<|z| \leq|x|} U_{x}^{-}(\mathrm{d} z)} \\
= & \lim _{r \uparrow|x|} \frac{\mathbb{E}_{x}\left[f \left(X_{\left.\left.\tau_{r}^{\oplus}\right) ; \tau_{r}^{\oplus}<\infty\right]}\right.\right.}{\mathbb{P}_{x}\left(\tau_{r}^{\oplus}=\infty\right)} .
\end{aligned}
$$

Substituting in the analytical form of the ratio on the right-hand side above using Eqs. 5.3 and 1.9 , we may continue with

$$
\begin{aligned}
& \mathbb{N}_{\arg (x)}\left(f\left(|x| e^{\epsilon(\zeta)} \Theta^{\epsilon}(\zeta)\right) ; \zeta<\infty\right) \\
= & \lim _{r \uparrow|x|} \pi^{-d / 2} \frac{\Gamma((d-\alpha) / 2)}{\Gamma(1-\alpha / 2)} \frac{\left.\left.\left(|x|^{2}-r^{2}\right)^{\alpha / 2} \int_{|y|<r}\left|r^{2}-\right| y\right|^{2}\right|^{-\alpha / 2}|x-y|^{-d} f(y) \mathrm{d} y}{\int_{0}^{\left(|x|^{2}-r^{2}\right) / r^{2}}(u+1)^{-d / 2} u^{\alpha / 2-1} \mathrm{~d} u} \\
= & \left.\pi^{-d / 2} \frac{\Gamma((d-\alpha) / 2)}{\Gamma(1-\alpha / 2)} \int_{|y|<|x|}|| x\right|^{2}-\left.|y|^{2}\right|^{-\alpha / 2}|x-y|^{-d} f(y) \mathrm{d} y \\
& \times \lim _{r \uparrow|x|} \frac{r^{\alpha}\left[\left(|x|^{2}-r^{2}\right) / r^{2}\right]^{\alpha / 2}}{\int_{0}^{\left(|x|^{2}-r^{2}\right) / r^{2}}(u+1)^{-d / 2} u^{\alpha / 2-1} \mathrm{~d} u} \\
= & \left.\frac{\alpha \pi^{-d / 2}}{2} \frac{\Gamma((d-\alpha) / 2)}{\Gamma(1-\alpha / 2)} \int_{|y|<|x|}|x|^{\alpha}|| x\right|^{2}-\left.|y|^{2}\right|^{-\alpha / 2}|x-y|^{-d} f(y) \mathrm{d} y,
\end{aligned}
$$

where we have used that the support of $f$ is compactly embedded in the ball of radius $|x|$ to justify the first term in the second equality.

Next we turn our attention to the quantity (5.1). Once again, our approach will be to scale an appropriate fluctuation identity by $\mathbb{P}_{x}\left(\tau_{r}^{\oplus}=\infty\right)=\int_{r<|z|<|x|} U_{x}^{-}(\mathrm{d} z)$. In this case, the natural object to work with is the expected occupation measure until first entry into the ball of radius $r<|x|$, where $x$ is the point of issue of the stable process. More precisely, we are interested in the resolvent density $h_{r}^{\oplus}(x, z),|x|,|z|>r$, which satisfies

$$
\int_{|z|>r} f(z) h_{r}^{\oplus}(x, z) \mathrm{d} z=\mathbb{E}_{x}\left[\int_{0}^{\tau_{r}^{\oplus}} f\left(X_{s}\right) \mathrm{d} s\right],
$$

for all measurable $f: \mathbb{R}^{d} \mapsto[0, \infty)$ with compact support. The aforesaid resolvent is not difficult to derive by applying the Riesz-Bogdan-Żak transform to Eq. 1.10. Indeed, Theorem 2 of [4] states that

$$
h_{r}^{\oplus}(x, z)=|x|^{\alpha-d}|z|^{\alpha-d} h_{1}^{\ominus}(K x, K z), \quad|x|,|z|>1 .
$$

Appealing to Eq. 4.5 it is now a straightforward exercise to deduce the following identity, known in the common potential analysis folklore, which we formally record for the sake of convenience. 
Corollary 5.1 (of Theorem 2 in [4]) For $|x|>r$, the resolvent (5.5) has a density given by

$$
h_{r}^{\oplus}(x, z)=2^{-\alpha} \pi^{-d / 2} \frac{\Gamma(d / 2)}{\Gamma(\alpha / 2)^{2}}|x-z|^{\alpha-d} \int_{0}^{\zeta_{r}^{\oplus}(x, z)}(u+1)^{-d / 2} u^{\alpha / 2-1} \mathrm{~d} u,
$$

where $\zeta_{r}^{\oplus}(x, z):=\left(|x|^{2}-r^{2}\right)\left(|z|^{2}-r^{2}\right) / r^{2}|x-z|^{2}$.

We can now use the above lemma to compute occupation potential with respect to the excursion measure. As for other results in this development, the following result is reminiscent of a classical result in fluctuation theory of Lévy processes, see e.g. exercise 5 in Chapter VI in [2], but as it includes the information about the modulator there is no direct way to derive it from the classical result.

Proposition 5.2 For $x \in \mathbb{R}^{d} \backslash\{0\}$, and continuous $g: \mathbb{R}^{d} \mapsto \mathbb{R}$ whose support is compactly embedded in the exterior of the ball of radius $|x|$,

$$
\mathbb{N}_{\arg (x)}\left(\int_{0}^{\zeta} g\left(|x| \mathrm{e}^{\epsilon(u)} \Theta^{\epsilon}(u)\right) \mathrm{d} u\right)=2^{-\alpha} \frac{\Gamma((d-\alpha) / 2)^{2}}{\Gamma(d / 2)^{2}} \int_{|x|<|z|} g(z) U_{x}^{+}(\mathrm{d} z)
$$

Proof Fix $0<r<|x|$. Recall from the Lamperti-Kiu representation (1.11) that $X_{t}=$ $\exp \left\{\xi_{\varphi(t)}\right\} \Theta(\varphi(t)), t \geq 0$, where $\int_{0}^{\varphi(t)} \exp \left\{\alpha \xi_{u}\right\} \mathrm{d} u=t$. In particular, this implies that, if we write $s=\varphi(t)$, then

$$
\mathrm{e}^{\alpha \xi_{s}} \mathrm{~d} s=\mathrm{d} t, \quad t>0 .
$$

Splitting the occupation over individual excursions, we have with the help of Eq. 2.1 that

$$
\begin{aligned}
& \mathbb{E}_{x}\left[\int_{0}^{\tau_{r}^{\oplus}} g\left(X_{t}\right) \mathrm{d} t\right] \\
= & \mathbb{E}_{x}\left[\int_{0}^{\infty} \mathbf{1}\left(\mathrm{e}^{\underline{\xi}} s>r\right) g\left(\mathrm{e}^{\xi_{s}} \Theta_{s}\right) \mathrm{e}^{\alpha \xi_{s}} \mathrm{~d} s\right] \\
= & \int_{r<|z|<|x|} U_{x}^{-}(\mathrm{d} z) \mathbb{N}_{\arg (z)}\left(\int_{0}^{\zeta} g\left(|z| \mathrm{e}^{\epsilon(s)} \Theta^{\epsilon}(s)\right)\left(|z| \mathrm{e}^{\epsilon(s)}\right)^{\alpha} \mathrm{d} s\right) .
\end{aligned}
$$

Note that the left-hand side is necessarily finite as it can be upper bounded by $\mathbb{E}_{x}\left[\int_{0}^{\infty} g\left(X_{t}\right) \mathrm{d} t\right]$, which is known to be finite for the given assumptions on $g$. Straightforward arguments, similar to those presented around (5.3), tell us that for continuous $g$ with compact support that is compactly embeded in the exterior of ball of radius $|x|$, we have that, for $r<|z|<|x|$,

$$
\mathbb{N}_{\arg (z)}\left(\int_{0}^{\zeta} g\left(|z| \mathrm{e}^{\epsilon(s)} \Theta^{\epsilon}(s)\right) \mathrm{e}^{\alpha \epsilon(s)} \mathrm{d} s\right)=\int_{0}^{\infty} \mathbb{N}_{\arg (z)}\left(g\left(|z| \mathrm{e}^{\epsilon(s)} \Theta^{\epsilon}(s)\right) \mathrm{e}^{\alpha \epsilon(s)} ; s<\zeta\right) \mathrm{d} s
$$


is a continuous function. Accordingly we can again use Lemma 3.1 and Theorem 1.2 and write, for $x \in \mathbb{R}^{d}$,

$$
\begin{aligned}
& \mathbb{N}_{\arg (x)}\left(\int_{0}^{\zeta} g\left(|x| \mathrm{e}^{\epsilon(s)} \Theta^{\epsilon}(s)\right)\left(|x| \mathrm{e}^{\epsilon(s)}\right)^{\alpha}\right) \\
= & \lim _{r \uparrow|x|} \frac{\int_{r<|z|<|x|} U_{x}^{-}(\mathrm{d} z) \mathbb{N}_{\arg (z)}\left(\int_{0}^{\zeta} g\left(|z| \mathrm{e}^{\epsilon(s)} \Theta^{\epsilon}(s)\right)\left(|z| \mathrm{e}^{\epsilon(s)}\right)^{\alpha}\right)}{\int_{r<|z| \leq|x|} U_{x}^{-}(\mathrm{d} z)} \\
= & \frac{\mathbb{E}_{x}\left[\int_{0}^{\tau_{r}^{\oplus}} g\left(X_{s}\right) \mathrm{d} s\right]}{\mathbb{P}_{x}\left(\tau_{r}^{\oplus}=\infty\right)} \\
= & 2^{-\alpha} \pi^{-d / 2} \frac{\Gamma((d-\alpha) / 2)}{\Gamma(\alpha / 2)} \lim _{r \uparrow|x|} \frac{\int_{|x|<|z|} \mathrm{d} z \mathbf{1}(r<|z|) g(z)|x-z|^{\alpha-d} \int_{0}^{\zeta_{r}^{\oplus}(x, z)}(u+1)^{-d / 2} u^{\alpha / 2-1} \mathrm{~d} u}{\int_{0}^{\left(|x|^{2}-r^{2}\right) / r^{2}}(u+1)^{-d / 2} u^{\alpha / 2-1} \mathrm{~d} u} \\
= & 2^{-\alpha} \pi^{-d / 2} \frac{\Gamma((d-\alpha) / 2)}{\Gamma(\alpha / 2)} \int_{|x|<|z|} \mathrm{d} z g(z)|x-z|^{-d}\left(|z|^{2}-|x|^{2}\right)^{\alpha / 2},
\end{aligned}
$$

where in the final equality we have used dominated convergence (in particular the assumption on the support of $g$ ). By inspection, we also note that the right-hand side above is equal to

$$
2^{-\alpha} \frac{\Gamma((d-\alpha) / 2)^{2}}{\Gamma(d / 2)^{2}} \int_{|x|<|z|} g(z)|z|^{\alpha} U_{x}^{+}(\mathrm{d} z)
$$

The proof is completed by replacing $g(z)$ by $g(z)|z|^{-\alpha}$.

\section{On $n$-Tuple Laws}

We are now ready to prove Theorems 1.3 and 1.4 and Corollary 1.3 with the help of Section 5 and other identities. In essence, we can piece together the desired results using Maisonneuve's exit formula (2.1) applied in the appropriate way, together with some of the identities established in previous section.

Proof of Theorem 1.3 (i) Appealing to the fact that the stable process $|X|$ does not creep downward and the Lévy system compensation formula for the jumps of $X$, we have, on the one hand,

$$
\mathbb{E}_{x}\left[f\left(X_{\mathrm{G}\left(\tau_{r}^{\oplus}\right)}\right) g\left(X_{\tau_{r}^{\oplus}-}\right) h\left(X_{\tau_{r}^{\oplus}}\right) ; \tau_{r}^{\oplus}<\infty\right]=\mathbb{E}_{x}\left[\int_{0}^{\tau_{r}^{\oplus}} f\left(X_{\mathrm{G}(t)}\right) g\left(X_{t}\right) k\left(X_{t}\right) \mathrm{d} t\right],
$$

where continuous $R$-valued functions $f, g, h$ are such that the first two are compactly supported in $\left\{z \in \mathbb{R}^{d}:|z|>r\right\}$ and the third is compactly supported in the open ball of radius $r$ and

$$
k(y)=\int_{|y+w|<r} \Pi(\mathrm{d} w) h(y+w) .
$$


On the other hand, a calculation similar in spirit to Eqs. 5.8, using 2.1, followed by an application of Proposition 5.2, tells us that

$$
\begin{aligned}
& \mathbb{E}_{x}\left[\int_{0}^{\tau_{r}^{\oplus}} f\left(X_{\mathrm{G}(t)}\right) g\left(X_{t}\right) k\left(X_{t}\right) \mathrm{d} t\right] \\
= & \int_{r<|z|<|x|} U_{x}^{-}(\mathrm{d} z) f(z) \mathbb{N}_{\arg (z)}\left(\int_{0}^{\zeta} g\left(|z| \mathrm{e}^{\epsilon(s)} \Theta^{\epsilon}(s)\right) k\left(|z| \mathrm{e}^{\epsilon(s)} \Theta^{\epsilon}(s)\right)\left(|z| \mathrm{e}^{\epsilon(s)}\right)^{\alpha} \mathrm{d} s\right) \\
= & 2^{-\alpha} \frac{\Gamma((d-\alpha) / 2)^{2}}{\Gamma(d / 2)^{2}} \int_{r<|z|<|x|} U_{x}^{-}(\mathrm{d} z) f(z) \int_{|z|<|y|} U_{z}^{+}(\mathrm{d} y) g(y) k(y)|y|^{\alpha} .
\end{aligned}
$$

Putting the pieces together, we get

$$
\begin{aligned}
& \mathbb{E}_{x}\left[f\left(X_{\mathrm{G}\left(\tau_{r}^{\oplus}\right)}\right) g\left(X_{\tau_{r}^{\oplus}}\right) h\left(X_{\tau_{r}^{\oplus}}\right) ; \tau_{r}^{\oplus}<\infty\right] \\
= & 2^{-\alpha} \frac{\Gamma((d-\alpha) / 2)^{2}}{\Gamma(d / 2)^{2}} \int_{r<|z|<|x|} \int_{|z|<|y|} \int_{|w-y|<r} U_{x}^{-}(\mathrm{d} z) U_{z}^{+}(\mathrm{d} y) \Pi(\mathrm{d} w) f(z) g(y)|y|^{\alpha} h(y+w) \\
= & c_{\alpha, d} \int_{r<|z|<|x|} \int_{|z|<|y|} \int_{|w-y|<r} \frac{\left.|| z\right|^{2}-\left.\left.|x|^{2}\right|^{\alpha / 2}|| y\right|^{2}-\left.|z|^{2}\right|^{\alpha / 2}}{|z|^{\alpha}|z-x|^{d}|z-y|^{d}|w|^{\alpha+d}} \mathrm{~d} y \mathrm{~d} z \mathrm{~d} w f(z) g(y) h(y+w) \\
= & c_{\alpha, d} \int_{r<|z|<|x|} \int_{|z|<|y|} \int_{|v|<r} \frac{\left.|| z\right|^{2}-\left.\left.|x|^{2}\right|^{\alpha / 2}|| y\right|^{2}-\left.|z|^{2}\right|^{\alpha / 2}}{|z|^{\alpha}|z-x|^{d}|z-y|^{d}|v-y|^{\alpha+d}} \mathrm{~d} y \mathrm{~d} z \mathrm{~d} v f(z) g(y) h(v)
\end{aligned}
$$

where

$$
c_{\alpha, d}=\frac{\Gamma((d+\alpha) / 2)}{|\Gamma(-\alpha / 2)|} \frac{\Gamma(d / 2)^{2}}{\pi^{3 d / 2} \Gamma(\alpha / 2)^{2}} .
$$

This is equivalent to the statement of part (i) of the theorem.

(ii) This is a straightforward application of the Riesz-Bogdan-Żak transformation, with computations in the style of those used to prove Corollary 5.1. For the sake of brevity, the proof is left as an exercise for the reader.

Proof of Corollary 1.3 As above, we only prove (i) as part (ii) can be derived appealing to the Riesz-Bogdan-Żak transformation.

From Eqs. 2.5, 4.3 and Proposition 5.1, more specifically (5.4), we have that for bounded measurable functions $f, g$ on $\mathbb{R}^{d}$,

$$
\begin{aligned}
& \mathbb{E}_{x}\left[g ( X _ { G ( \tau _ { 1 } ^ { \oplus } ) } ) f \left(X_{\left.\left.\tau_{1}^{\oplus}\right) ; \tau_{1}^{\oplus}<\infty\right]}\right.\right. \\
= & \int_{1<|z|<|x|} U_{x}^{-}(\mathrm{d} z) \mathbb{N}_{\arg (z)}\left(f\left(|z| e^{\epsilon(\zeta)} \Theta^{\epsilon}(\zeta)\right) \mathbf{1}\left(|z| e^{\epsilon(\zeta)}<1\right) ; \zeta<\infty\right) g(z) \\
= & \frac{\Gamma(d / 2)^{2} \sin (\pi \alpha / 2)}{\pi^{d}|\Gamma(-\alpha / 2)| \Gamma(\alpha / 2)} \int_{1<|z|<|x|} \int_{|v|<1} \frac{\left.|| z\right|^{2}-\left.|x|^{2}\right|^{\alpha / 2}}{\left.|| z\right|^{2}-\left.|v|^{2}\right|^{\alpha / 2}|z-v|^{d}|z-x|^{d}} f(v) g(z) \mathrm{d} z \mathrm{~d} v .
\end{aligned}
$$

This gives the desired result when $r=1$. As usual, we use scaling to convert the above conclusion to the setting of first passage into a ball of radius $r>0$. 
Proof of Corollary 1.4 As with the previous proof, we only deal with (i) and the case that $r=1$ for the same reasons. Setting $f \equiv 1$ in Eq. 6.1, we see with the help of Corollary 5.1 and Eq. 1.1 that

$$
\begin{aligned}
& \mathbb{E}_{x}\left[g\left(X_{\tau_{1}^{\oplus}}\right) h\left(X_{\tau_{1}^{\oplus}}\right) ; \tau_{1}^{\oplus}<\infty\right] \\
= & \mathbb{E}_{x}\left[\int_{0}^{\tau_{1}^{\oplus}} g\left(X_{t}\right) k\left(X_{t}\right) \mathrm{d} t\right] \\
= & \frac{2^{\alpha} \Gamma((d+\alpha) / 2)}{\pi^{d / 2}|\Gamma(-\alpha / 2)|} \int_{|y|>1} g(y) \int_{|y+w|<1} \frac{1}{|w|^{\alpha+d}} \mathrm{~d} w h(y+w) h_{1}^{\oplus}(x, y) \mathrm{d} y \\
= & \frac{2^{\alpha} \Gamma((d+\alpha) / 2)}{\pi^{d / 2}|\Gamma(-\alpha / 2)|} \int_{|y|>1} \int_{|v|<1} g(y) h(v) \frac{1}{|v-y|^{\alpha+d}} h_{1}^{\oplus}(x, y) \mathrm{d} v \mathrm{~d} y
\end{aligned}
$$

where the function $k(\cdot)$ is as before. The result now follows.

\section{Deep Factorisation of the Stable Process}

The manipulations we have made in Section 5, in particular in Proposition 5.2, are precisely what we need to demonstrate the Wiener-Hopf factorisation. Recall that, for Theorem 1.4, we defined

$$
\mathbf{R}_{z}[f](\theta)=\mathbf{E}_{0, \theta}\left[\int_{0}^{\infty} \mathrm{e}^{-z \xi_{t}} f\left(\Theta_{t}\right) \mathrm{d} t\right], \quad \theta \in \mathbb{S}^{d-1}, z \in \mathbb{C} .
$$

Moreover, define

$$
\hat{\boldsymbol{\rho}}_{z}[f](\theta)=\mathbf{E}_{0, \theta}\left[\int_{0}^{\infty} \mathrm{e}^{-z H_{t}^{-}} f\left(\Theta_{t}^{-}\right) \mathrm{d} t\right]=\int_{|y|<1}|y|^{z} f(\arg (y)) U_{\theta}^{-}(\mathrm{d} y)
$$

and

$$
\boldsymbol{\rho}_{z}[f](\theta)=\mathbf{E}_{0, \theta}\left[\int_{0}^{\infty} \mathrm{e}^{-z H_{t}^{+}} f\left(\Theta_{t}^{+}\right) \mathrm{d} t\right]=\int_{|y|>1}|y|^{-z} f(\arg (y)) U_{\theta}^{+}(\mathrm{d} y)
$$

for bounded measurable $f: \mathbb{S}^{d-1} \mapsto[0, \infty)$, whenever the integrals make sense. We note that the expression for $\boldsymbol{\rho}_{z}[f](\theta)$, as given in the statement of Theorem 1.4, is clear given Eq. 4.4. Moreover, from e.g. Section 2 of [3], it is known that the free potential measure of a stable process issued from $x \in \mathbb{R}^{d}$ has a density given by

$$
u(x, y)=\frac{\Gamma((d-\alpha) / 2)}{2^{\alpha} \pi^{d / 2} \Gamma(\alpha / 2)}|y-x|^{\alpha-d}, \quad y \in \mathbb{R}^{d} .
$$

Accordingly, taking account of Eq. 5.7, it is straightforward to formally compute

$$
\begin{aligned}
\mathbf{R}_{z}[f](\theta) & =\mathbf{E}_{0, \theta}\left[\int_{0}^{\infty} \mathrm{e}^{-(z+\alpha) \xi_{s}} f\left(\Theta_{s}\right) \mathrm{e}^{\alpha \xi_{s}} \mathrm{~d} s\right] \\
& =\mathbb{E}_{\theta}\left[\int_{0}^{\infty}\left|X_{t}\right|^{-(\alpha+z)} f\left(\arg \left(X_{t}\right)\right) \mathrm{d} t\right] \\
& =\int_{\mathbb{R}^{d}} f(\arg (y)) \frac{u(\theta, y)}{|y|^{\alpha+z}} \mathrm{~d} y, \quad \operatorname{Re}(z) \geq 0,
\end{aligned}
$$

where we have used stationary and independent increments in the final equality and both sides of Eq. 7.1 are understood to be infinite at the same time. We note, however, that the integral on the right-hand side of Eq. 7.1 is finite if and only if $\operatorname{Re}(z) \in(0, d-\alpha)$. 
Indeed, by changing variables from $y \in \mathbb{R}^{d}$ to $r \theta, r \geq 0$ and $\theta \in \mathbb{S}^{d-1}$, we have that the aforesaid integral is $O\left(\int_{0+} r^{d-\alpha-z-1} \mathrm{~d} r\right)$ when $y$ is near the origin, providing $\theta \neq 0$, of order $O\left(\int_{0+} r^{-z-1} \mathrm{~d} r\right)$ when $y$ is near the origin if $\theta=0$ and of order $O\left(\int^{\infty} r^{-z-1} \mathrm{~d} r\right)$ as $|y| \rightarrow \infty$.

Proof of Theorem 1.4 From the second and third equalities of Eq. 5.8 (taking $r \rightarrow 0$ ) and Proposition 5.2, providing the left-hand side is finite, which it is for $c \in(0, d-\alpha)$ and $\lambda \in \mathbb{R}$, we have

$$
\begin{aligned}
\mathbf{R}_{c-\mathrm{i} \lambda}[f](\theta) & =\int_{|w|<1} U_{\theta}^{-}(\mathrm{d} w) \mathbb{N}_{\arg (w)}\left(\int_{0}^{\zeta}\left(|w| \mathrm{e}^{\epsilon(s)}\right)^{-c+\mathrm{i} \lambda} f\left(\Theta^{\epsilon}(s)\right)\right) \\
& =2^{-\alpha} \frac{\Gamma((d-\alpha) / 2)^{2}}{\Gamma(d / 2)^{2}} \int_{|w|<1} U_{\theta}^{-}(\mathrm{d} w) \int_{|w|<|y|} f(\arg (y))|y|^{-c+\mathrm{i} \lambda} U_{w}^{+}(\mathrm{d} y) .
\end{aligned}
$$

Note that, by conditional stationary and independent increments, for any $w \in \mathbb{R}^{d} \backslash\{0\}$ and $c>0$,

$$
\begin{aligned}
\int_{|w|<|y|}|y|^{-c+\mathrm{i} \lambda} f(\arg (y)) U_{w}^{+}(\mathrm{d} y) & =\mathbf{E}_{\log |w|, \arg (w)}\left[\int_{0}^{\infty} \mathrm{e}^{-c+\mathrm{i} \lambda H_{t}^{+}} f\left(\Theta_{t}^{+}\right) \mathrm{d} t\right] \\
& =|w|^{-c+\mathrm{i} \lambda} \mathbf{E}_{0, \arg (w)}\left[\int_{0}^{\infty} \mathrm{e}^{-c+\mathrm{i} \lambda H_{t}^{+}} f\left(\Theta_{t}^{+}\right) \mathrm{d} t\right] \\
& =|w|^{-c+\mathrm{i} \lambda} \int_{1<|y|}|y|^{-c+\mathrm{i} \lambda} f(\arg (y)) U_{\arg (w)}^{+}(\mathrm{d} y),
\end{aligned}
$$

where, by Eq. 4.4, converting to polar coordinates, the right-hand side is uniformly bounded by an integral of $O\left(\int^{\infty} r^{-c-1} \mathrm{~d} r\right)<\infty$. Hence plugging (7.3) back in Eq. 7.2, we have

$$
\mathbf{R}_{c-\mathrm{i} \lambda}[f](\theta)=2^{-\alpha} \frac{\Gamma((d-\alpha) / 2)^{2}}{\Gamma(d / 2)^{2}} \hat{\boldsymbol{\rho}}_{c-\mathrm{i} \lambda}\left[\boldsymbol{\rho}_{c-\mathrm{i} \lambda}[f]\right](\theta), \quad \lambda \in \mathbb{R}, c \in(0, d-\alpha) .
$$

Finally, we note from Eq. 4.4 that, making the change of variables $y=K w$, so that $\arg (y)=$ $\arg (w),|y|=1 /|w|$ and $\mathrm{d} y=|w|^{-2 d} \mathrm{~d} w$ and, for $\theta \in \mathbb{S}^{d-1},|\theta-K w|=|\theta-w| /|w|$, we have

$$
\begin{aligned}
\hat{\boldsymbol{\rho}}_{c-\mathrm{i} \lambda}[f](\theta) & =\pi^{-d / 2} \frac{\Gamma(d / 2)^{2}}{\Gamma((d-\alpha) / 2) \Gamma(\alpha / 2)} \int_{|y|>1}|y|^{-c+\mathrm{i} \lambda} f(\arg (y)) \frac{\left.|| y\right|^{2}-\left.1\right|^{\alpha / 2}}{|y|^{\alpha}|\theta-y|^{d}} \mathrm{~d} y \\
& =\pi^{-d / 2} \frac{\Gamma(d / 2)^{2}}{\Gamma((d-\alpha) / 2) \Gamma(\alpha / 2)} \int_{1<|w|}|w|^{c-\mathrm{i} \lambda+\alpha-d} f(\arg (w)) \frac{\left.\left.|1-| w\right|^{2}\right|^{\alpha / 2}}{|\theta-w|^{d}|w|^{\alpha}} \mathrm{d} w \\
& =\rho_{\mathrm{i} \lambda+d-\alpha-c}[f](\theta), \quad \lambda \in \mathbb{R}, c \in(0, d-\alpha)
\end{aligned}
$$

which is also uniformly bounded by an integral of $O\left(\int^{\infty} r^{c-(d-\alpha)-1} \mathrm{~d} r\right)<\infty$.

\section{Proof of Theorem 1.5}

Recall from the description of the Riesz-Bogdan-Żak transform that $(\xi, \Theta)$ under the change of measure in Eq. 4.2 is equal in law to $(-\xi, \Theta)$. Accordingly, we have for $q>0$, 
$x \in \mathbb{R}^{d} \backslash\{0\}$ and bounded measurable $g$ whose support is compactly embedded in the ball of unit radius,

$$
\begin{aligned}
& \mathbf{E}_{-\log |x|, \arg (x)}\left[g\left(\mathrm{e}^{-\left(\bar{\xi}_{\mathbf{\xi}_{q}}-\xi_{\mathbf{\xi}_{q}}\right)} \Theta_{\mathbf{e}_{q}}\right)\right] \\
= & \mathbf{E}_{\log |x|, \arg (x)}\left[\frac{\mathrm{e}^{(\alpha-d) \xi_{\mathbf{e}_{q}}}}{|x|^{\alpha-d}} g\left(\mathrm{e}^{-\left(\xi_{\mathbf{e}_{q}}-\xi_{\mathbf{e}_{q}}\right)} \Theta_{\mathbf{e}_{q}}\right)\right] \\
= & |x|^{d-\alpha} \mathbf{E}_{\log |x|, \arg (x)}\left[\sum_{g \in G} \mathbf{1}\left(\zeta_{g^{\prime}}<\mathbf{e}_{q}^{g^{\prime}}, \forall G \ni \mathrm{g}^{\prime}<g\right) \mathrm{e}^{(\alpha-d) \xi_{g}} \mathrm{e}^{(\alpha-d) \epsilon_{g}\left(\mathbf{e}_{q}^{g}\right)} g\left(\mathrm{e}^{-\epsilon_{g}\left(\mathbf{e}_{q}^{g}\right)} \Theta_{g}^{\epsilon}\left(\mathbf{e}_{q}^{g}\right)\right) \mathbf{1}\left(\mathbf{e}_{q}^{\mathrm{g}}<\zeta_{g}\right)\right] \\
= & |x|^{d-\alpha} \mathbf{E}_{\log |x|, \arg (x)}\left[\int_{0}^{\infty} \mathrm{e}^{-q t} \mathrm{e}^{(\alpha-d) \xi_{t}} \mathbb{N}_{\Theta_{t}}\left(\mathrm{e}^{(\alpha-d) \epsilon\left(\mathbf{e}_{q}\right)} g\left(\mathrm{e}^{-\epsilon\left(\mathbf{e}_{q}\right)} \Theta\left(\mathbf{e}_{q}\right)\right) ; \mathbf{e}_{q}<\zeta\right) \mathrm{d} L_{t}\right] \\
= & |x|^{d-\alpha} \mathbf{E}_{\log |x|, \arg (x)}\left[\int_{0}^{\infty} \mathrm{e}^{-q \ell_{s}^{-1}} \mathrm{e}^{-(\alpha-d) H_{s}^{-}} \mathbb{N}_{\Theta_{s}^{-}}\left(\mathrm{e}^{(\alpha-d) \epsilon\left(\mathbf{e}_{q}\right)} g\left(\mathrm{e}^{-\epsilon\left(\mathbf{e}_{q}\right)} \Theta\left(\mathbf{e}_{q}\right)\right) ; \mathbf{e}_{q}<\zeta\right) \mathrm{d} s\right],
\end{aligned}
$$

where, for each $g \in G, \mathbf{e}_{q}^{g}$ are additional marks on the associated excursion which are independent and exponentially distributed with rate $q$. Hence, if we define

$$
U_{x}^{(q),-}(\mathrm{d} y)=\int_{0}^{\infty} \mathrm{d} s \mathbf{E}_{\log |x|, \arg (x)}\left[\mathrm{e}^{-q \ell_{t}^{-1}} ; \mathrm{e}^{-H_{s}^{-}} \Theta_{s}^{-} \in \mathrm{d} y, s<\ell_{\infty}\right], \quad|y|<|x|,
$$

then

$$
\begin{aligned}
& \mathbf{E}_{-\log |x|, \arg (x)}\left[g\left(\mathrm{e}^{-\left(\bar{\xi}_{\mathbf{e}_{q}}-\xi_{\mathbf{e}_{q}}\right)} \Theta_{\mathbf{e}_{q}}\right)\right] \\
= & \int_{(0, \infty)} \int_{|y|<|x|} q U_{x}^{(q),-}(\mathrm{d} y) \frac{|y|^{\alpha-d}}{|x|^{\alpha-d}} \mathbb{N}_{\arg (y)}\left(\int_{0}^{\zeta} \mathrm{e}^{-q t} \mathrm{e}^{(\alpha-d) \epsilon(t)} g\left(\mathrm{e}^{-\epsilon(t)} \Theta(t)\right) \mathrm{d} t\right) .
\end{aligned}
$$

Recall that $\ell^{-1}$ is a subordinator (without reference to the accompanying modulation $\left.\Theta^{+}\right)$, suppose we denote its Laplace exponent by $\Lambda^{+}(q):=-\log \mathbf{E}_{0, \theta}\left[\exp \left\{-q L_{1}^{-1}\right\}\right]$, $q \geq 0$, where $\theta \in \mathbb{S}^{d-1}$ is unimportant in the definition. Appealing again to the RieszBogdan-Żak transform again, we also note that for a bounded and measurable function $h$ on $\mathbb{S}^{d-1}$, using obvious notation

$$
\begin{aligned}
\int_{|y|<|x|} \frac{|y|^{\alpha-d}}{|x|^{\alpha-d}} q U_{x}^{(q),-}(\mathrm{d} y) h(\arg (y)) & =q \int_{0}^{\infty} \mathrm{d} s \mathbf{E}_{-\log |x|, \arg (x)}\left[\mathrm{e}^{-q L_{t}^{-1}} h\left(\Theta_{s}^{+}\right)\right] \\
& =\frac{q}{\Lambda^{+}(q)} \int_{0}^{\infty} \mathrm{d} s \Lambda^{+}(q) \mathrm{e}^{-\Lambda^{+}(q) s} \mathbf{E}_{-\log |x|, \arg (x)}^{(q)}\left[h\left(\Theta_{s}^{+}\right)\right] \\
& =\frac{q}{\Lambda^{+}(q)} \mathbf{E}_{-\log |x|, \arg (x)}^{(q)}\left[h\left(\Theta_{\mathbf{e}_{\Lambda^{+}(q)}^{+}}\right)\right]
\end{aligned}
$$

where $\mathbf{P}_{-\log |x|, \arg (x)}^{(q)}$ appears as the result of a change of measure with martingale density $\exp \left\{-q L_{s}^{-1}+\Lambda^{+}(q) s\right\}, s \geq 0$, and $\Lambda^{+}(q)$ is the Laplace exponent of the subordinator $L^{-1}$ and $\mathbf{e}_{\Lambda^{+}(q)}$ is an independent exponential random variable with parameter $\Lambda^{+}(q)$.

Next, we want to take $q \downarrow 0$ in Eq. 8.1. To this end, we start by remarking that, as $L$ is a local time for the Lévy process $\xi$ (without reference to its modulation), it is known from classical Wiener-Hopf factorisation theory that, up to a multiplicative constant, $c>$ 0 , which depends on the normalisation of the local time $L, q=c \Lambda^{+}(q) \Lambda^{-}(q)$, where $\Lambda^{-}(q)$ is the Laplace exponent of the local time at the infimum $\ell$; see for example equation (3) in Chapter VI of [2]. On account of the fact that $X$ is transient, we know that $\ell_{\infty}$ is 
exponentially distributed and the reader may recall that we earlier normalised our choice of $\ell$ such that its rate, $\Lambda^{-}(0)=1$. This implies, in turn, that $\lim _{q \downarrow 0} q / \Lambda^{+}(q)=c$.

Appealing to isotropy, the recurrence of $\{0\} \times \mathbb{S}^{d-1}$ for $(\bar{\xi}-\xi, \Theta)$ and weak convergence back in Eq. 8.2 as we take the limit with $q \downarrow 0$, to find that

$$
\lim _{q \rightarrow 0} \int_{|y|<|x|} \frac{|y|^{\alpha-d}}{|x|^{\alpha-d}} q U_{x}^{(q),-}(\mathrm{d} y) h(\arg (y))=c \int_{\mathbb{S}^{d-1}} \sigma_{1}(\mathrm{~d} \phi) h(\phi),
$$

where we recall that $\sigma_{1}(\mathrm{~d} \phi)$ is the surface measure on $\mathbb{S}^{d-1}$ normalised to have unit mass. Hence, back in Eq. 8.1 we have with the help of Proposition 5.2 and Eq. 4.4,

$$
\begin{aligned}
& \lim _{q \downarrow 0} \mathbf{E}_{-\log |x|, \arg (x)}\left[g\left(\mathrm{e}^{-\left(\bar{\xi}_{\mathbf{e}_{q}}-\xi_{\mathbf{e}_{q}}\right)} \Theta_{\mathbf{e}_{q}}\right)\right] \\
= & \int_{\mathbb{S}^{d-1}} \sigma_{1}(\mathrm{~d} \phi) \mathbb{N}_{\phi}\left(\int_{0}^{\zeta} \mathrm{e}^{(\alpha-d) \epsilon(t)} g\left(\mathrm{e}^{-\epsilon(t)} \Theta(t)\right) \mathrm{d} t\right) \\
= & c \pi^{-d / 2} 2^{-\alpha} \frac{\Gamma((d-\alpha) / 2)}{\Gamma(\alpha / 2)} \int_{\mathbb{S}^{d-1}} \sigma_{1}(\mathrm{~d} \phi) \int_{1<|z|} g(K z) \frac{\left.|| z\right|^{2}-\left.1\right|^{\alpha / 2}}{|z|^{d}|\phi-z|^{d}} \mathrm{~d} z,
\end{aligned}
$$

where we recall that $K z=z /|z|^{2}$. Finally, we note that, using the Lamperti-Kiu transform and Eq. 5.7, for bounded measurable $f$ and compactly embedded in $\mathbb{B}_{d}$,

$$
f\left(X_{t} / M_{t}\right) \mathrm{d} t=f\left(\mathrm{e}^{-\left(\bar{\xi}_{s}-\xi_{s}\right)} \Theta_{s}\right) \mathrm{e}^{\alpha \xi_{s}} \mathrm{~d} s
$$

where $s=\varphi(t)$, suggesting that, for $y \in \mathbb{R}^{d} \backslash\{0\}$,

$$
\lim _{t \rightarrow \infty} \mathbb{E}_{y}\left[f\left(X_{t} / M_{t}\right)\right]=\lim _{s \rightarrow \infty} \mathbf{E}_{\log |y|, \arg (y)}\left[f\left(\mathrm{e}^{-\left(\bar{\xi}_{s}-\xi_{s}\right)} \Theta_{s}\right) \mathrm{e}^{\alpha \xi_{s}}\right] .
$$

In fact, we can justify this rigorously appealing to the discussion at the bottom of $\mathrm{p} 240$ of [20]. Hence, putting this together with Eq. 8.3, for $f$ and $x$ as before, we conclude that,

$$
\begin{aligned}
\lim _{t \rightarrow \infty} \mathbb{E}_{K x}\left[f\left(X_{t} / M_{t}\right)\right] & =\lim _{q \downarrow 0} \mathbf{E}_{-\log |x|, \arg (x)}\left[f\left(\mathrm{e}^{-\left(\bar{\xi}_{\mathbf{e}_{q}}-\xi_{\mathbf{e}_{q}}\right)} \Theta_{\mathbf{e}_{q}}\right) \mathrm{e}^{\alpha \xi_{\mathbf{e}_{q}}}\right] \\
& =c \pi^{-d / 2} 2^{-\alpha} \frac{\Gamma((d-\alpha) / 2)}{\Gamma(\alpha / 2)} \int_{\mathbb{S}^{d-1}} \sigma_{1}(\mathrm{~d} \phi) \int_{1<|z|} f(K z) \frac{\left.|K z|^{\alpha}|| z\right|^{2}-\left.1\right|^{\alpha / 2}}{|z|^{d}|\phi-z|^{d}} \mathrm{~d} z \\
& =c \pi^{-d / 2} 2^{-\alpha} \frac{\Gamma((d-\alpha) / 2)}{\Gamma(\alpha / 2)} \int_{\mathbb{S}^{d-1}} \sigma_{1}(\mathrm{~d} \phi) \int_{|w|<1} f(w) \frac{\left.\left.|1-| w\right|^{2}\right|^{\alpha / 2}}{|\phi-w|^{d}} \mathrm{~d} w
\end{aligned}
$$

where we changed variables $w=K z$, or equivalently $z=K w$, and we used Eq. 4.5 , that $|w|=1 /|z|$ and that $\mathrm{d} z=\mathrm{d} w /|w|^{2 d}$.

In order to pin down the constant $c$, we need to ensure that, when $f \equiv 1$, the integral on the right-hand side of Eq. 8.4 is identically equal to 1 . To do this, we recall a classical Poisson potential formula (see for example Theorem 4.3.1 in [18])

$$
\left(1-|w|^{2}\right)^{-1}=\int_{\mathbb{S}^{d-1}}|\phi-w|^{-d} \sigma_{1}(\mathrm{~d} \phi) \quad|w|<1 .
$$


Writing $\sigma_{r}(\mathrm{~d} \theta), \theta \in r \mathbb{S}^{d-1}$ for the uniform surface measure on $r \mathbb{S}^{d-1}$ normalised to have total mass equal to one, it follows that

$$
\begin{aligned}
\int_{\mathbb{S}^{d-1}} \sigma_{1}(\mathrm{~d} \phi) \int_{|w|<1} \frac{\left.\left.|1-| w\right|^{2}\right|^{\alpha / 2}}{|\phi-w|^{d}} \mathrm{~d} w & =\left.\left.\int_{|w|<1}|1-| w\right|^{2}\right|^{\frac{\alpha}{2}-1} \mathrm{~d} w \\
& =\frac{2 \pi^{d / 2}}{\Gamma(d / 2)} \int_{0}^{1} r^{d-1} \mathrm{~d} r \int_{r \mathbb{S}^{d-1}} \sigma_{r}(\mathrm{~d} \theta)\left(1-r^{2}\right)^{\frac{\alpha}{2}-1} \\
& =\frac{\pi^{d / 2}}{\Gamma(d / 2)} \int_{0}^{1} y^{\frac{d}{2}-1}(1-y)^{\frac{\alpha}{2}-1} \mathrm{~d} y \\
& =\pi^{d / 2} \frac{\Gamma(\alpha / 2)}{\Gamma((d+\alpha) / 2)} .
\end{aligned}
$$

This forces us to take

and so,we have

$$
c=2^{\alpha} \frac{\Gamma((d+\alpha) / 2)}{\Gamma((d-\alpha) / 2)}
$$

$$
\lim _{t \rightarrow \infty} \mathbb{E}_{K x}\left[f\left(X_{t} / M_{t}\right)\right]=\pi^{-d / 2} \frac{\Gamma((d+\alpha) / 2)}{\Gamma(\alpha / 2)} \int_{\mathbb{S}^{d-1}} \sigma_{1}(\mathrm{~d} \phi) \int_{|w|<1} f(w) \frac{\left.\left.|1-| w\right|^{2}\right|^{\alpha / 2}}{|\phi-w|^{d}} \mathrm{~d} w .
$$

as required.

Acknowledgements The authors would like to thank Ron Doney who pointed out the distributional interpretations in Remarks 1.1 and 1.5. We would also like to thank two anonymous referees who provided two extremely helpful and thorough reports on an earlier version of this paper.

Open Access This article is distributed under the terms of the Creative Commons Attribution 4.0 International License (http://creativecommons.org/licenses/by/4.0/), which permits unrestricted use, distribution, and reproduction in any medium, provided you give appropriate credit to the original author(s) and the source, provide a link to the Creative Commons license, and indicate if changes were made.

\section{References}

1. Alili, L., Chaumont, L., Graczyk, P., Żak, T.: Inversion, duality and doob $h$-transforms for self-similar Markov processes. Electron. J. Probab., 22:Paper No. 20 (2017)

2. Bertoin, J.: Lévy Processes, Volume 121 of Cambridge Tracts in Mathematics. Cambridge University Press, Cambridge (1996)

3. Blumenthal, R.M., Getoor, R.K., Ray, D.B.: On the distribution of first hits for the symmetric stable processes. Trans. Amer. Math. Soc. 99, 540-554 (1961)

4. Bogdan, K., Żak, T.: On Kelvin transformation. J. Theoret. Probab. 19(1), 89-120 (2006)

5. Caballero, M.E., Pardo, J.C., Pérez, J.L.: Explicit identities for Lévy processes associated to symmetric stable processes. Bernoulli 17(1), 34-59 (2011)

6. Chaumont, L., Kyprianou, A.E., Pardo, J.C., Rivero, V.: Fluctuation theory and exit systems for positive self-similar Markov processes. Ann. Probab. 40(1), 245-279 (2012)

7. Chaumont, L., Pantí, H., Rivero, V.: The Lamperti representation of real-valued self-similar Markov processes. Bernoulli 19(5B), 2494-2523 (2013)

8. Kuznetsov, A., Kyprianou, A.E., Pardo, J.C.: Meromorphic Lévy processes and their fluctuation identities. Ann. Appl. Probab. 22(3), 1101-1135 (2012)

9. Kuznetsov, A., Kyprianou, A.E., Pardo, J.C., Watson, A.R.: The hitting time of zero for a stable process. Electron. J. Probab. 19(30), 26 (2014)

10. Kyprianou, A.E. Fluctuations of Lévy Processes with Applications. Universitext, 2nd edn. Springer, Heidelberg (2014). Introductory lectures

11. Kyprianou, A.E.: Deep factorisation of the stable process. Electron. J. Probab., 21:Paper No. 23 (2016)

12. Kyprianou, A.E., Pardo, J.C., Rivero, V.: Exact and asymptotic $n$-tuple laws at first and last passage. Ann. Appl. Probab. 20(2), 522-564 (2010) 
13. Kyprianou, A.E., Pardo, J.C., Watson, A.R.: Hitting distributions of $\alpha$-stable processes via path censoring and self-similarity. Ann. Probab. 42(1), 398-430 (2014)

14. Kyprianou, A.E., Rivero, V., Sengul, B.: Deep factorisation of the stable process II: potentials and applications. (with Victor M. Rivero and Bati Sengul). Annales de l'Instut Henri Poincaré 54(1), 343-362 (2018)

15. Kyprianou, A.E., Kyprianou, A.E., Rivero, V., Satitkanitkul, W.: Conditioned real self-similar Markov processes. Stochastic Processes and their Applications 127, 1234-1254 (2017)

16. Kyprianou, A.E.: Stable processes, self-similarity and the unit ball. arXiv:1707.04343 [math.PR] (2017)

17. Maisonneuve, B.: Exit systems. Ann. Probab. 3(3), 399-411 (1975)

18. Port, S.C., Stone, C.J.: Brownian Motion and Classical Potential Theory. Academic Press [Harcourt Brace Jovanovich Publishers], New York (1978). Probability and Mathematical Statistics

19. Schilling, R.L., Song, R., Vondraček, Z. Bernstein Functions Volume 37 of de Gruyter Studies in Mathematics, 2nd edn. Walter de Gruyter \& Co., Berlin (2012). Theory and applications

20. Walsh, J.B.: Markov processes and their functionals in duality. Z. Wahrscheinlichkeitstheorie und Verw. Gebiete 24, 229-246 (1972)

Publisher's Note Springer Nature remains neutral with regard to jurisdictional claims in published maps and institutional affiliations. 